\title{
Leibniz on Natural Teleology and the Laws of Optics
}

\section{Citation}

McDonough, Jeffrey K. 2009. Leibniz on natural teleology and the laws of optics. Philosophy and Phenomenological Research 78(3): 505-544.

\section{Published Version}

doi:10.1111/j.1933-1592.2009.00254.x

\section{Permanent link}

http://nrs.harvard.edu/urn-3:HUL.InstRepos:5130440

\section{Terms of Use}

This article was downloaded from Harvard University's DASH repository, and is made available under the terms and conditions applicable to Open Access Policy Articles, as set forth at http:// nrs.harvard.edu/urn-3:HUL.InstRepos:dash.current.terms-of-use\#OAP

\section{Share Your Story}

The Harvard community has made this article openly available.

Please share how this access benefits you. Submit a story.

Accessibility 


\title{
forthcoming Philosophy and Phenomenological Research ...
}

\section{Leibniz on Natural Teleology and the Laws of Optics}

\author{
Jeffrey K. McDonough \\ Department of Philosophy \\ Harvard University
}

\begin{abstract}
This essay examines one of the cornerstones of Leibniz's defense of teleology within the order of nature. The first section explores Leibniz's contributions to the study of geometrical optics, and argues that his introduction of the "Most Determined Path Principle" or "MDPP" allows him to bring to the fore philosophical issues concerning the legitimacy of teleological explanations by addressing two technical objections raised by Cartesians to non-mechanistic derivations of the laws of optics. The second section argues that, by drawing on laws such as the MDPP, Leibniz is able to introduce a thin notion of teleology that gives him the resources to respond to the most pressing charges of his day against teleological explanations within natural philosophy. Finally, the third section argues that contemporary philosophers have been overly hasty in their dismissal of Leibniz's account of natural teleology, and indeed that their own generally thin conceptions of teleology have left them with few well-motivated resources for resisting his elegant position.
\end{abstract}

\section{Introduction}

At a first pass, a teleological explanation is an explanation that attempts to explain a behavior or event by appealing to an outcome or consequence of that behavior or event. "Betty is going to law school in order to get rich;" "The bear is swatting at the beehive in 
order to get honey;" "The heat-seeking missile swerved left in order to hit its target;" and "Stones fall in order to reach their natural resting place at the center of the earth" are thus all at least candidates for teleological explanations. The likely or expected outcomes appealed to in putatively teleological explanations such as these are often called "goals," “ends," and, although less commonly today, "final causes."

It is well known that the use of teleological explanations in the domain of physics underwent a massive reevaluation during the early modern period. Prior to the scientific revolution, explanations in terms of final causes were widely accepted, and even thought to be necessary for an adequate account of the natural world. After the scientific revolution, teleological explanations were generally shunned in the practice of physics, and eventually became viewed with suspicion even in relation to biological phenomena. Understandably curious about this dramatic shift in explanatory mores, scholars of the early modern period have devoted a great deal of attention to Cartesian and Spinozistic critiques of the use of final causes, and have significantly increased our understanding of the grounds, implications, and limitations of those criticisms. ${ }^{1}$

It is also well-known that Leibniz was one of the most important early modern opponents of the wholesale rejection of teleology and teleological explanations as applied to the physical world. Although committed at least in broad terms to the project of the new science, Leibniz insists that final causes may be "useful not only for admiring the ingenuity of the great workman, but also for discovering something useful in physics and

\footnotetext{
${ }^{1}$ See, for example, Bennett (1983; 1984, 213-230; 1990), Curley (1990), Della Rocca (1996, 252-257), Gabbey (1980), Garrett (1999), Rice (1985), and Simmons (2001).
} 
medicine" (DM 22/FW 74). ${ }^{2}$ In spite of the remarkable contrast between Leibniz's position and the growing consensus of his day, however, there has been relatively little attention devoted to Leibniz's defense of teleology. This neglect is all the more striking given that Leibniz is much more interested in wrestling with issues surrounding teleology and teleological explanations than are Descartes and Spinoza. Whereas his two distinguished predecessors limit their discussions of final causes to a mere handful of passages, Leibniz returns to the topic with an almost obsessive regularity.

The present essay examines one of the cornerstones of Leibniz's defense of teleology within the order of nature, namely, his derivation of the two central laws of

${ }^{2}$ I use the following abbreviations for Leibniz's and Descartes's standard texts (full references are provided in the bibliography): $A=$ German Academy of Sciences (ed.) Gottfried Wilhelm Leibniz: Sämtliche Schriften und Briefe, 1926-; AG=R. Ariew and D. Garber (eds. and trans.) G. W. Leibniz: Philosophical Essays, 1989; AT=C. Adam and P. Tannery (eds.) Oeuvres des Descartes, 12 Volumes, 1964-76; CSM=J. Cottingham, R. Stoothoff, and D. Murdoch (eds.) The Philosophical Writings of Descartes, Volumes 1 and 2, 1985; DC= G. W. Leibniz, "Definitiones Cogitationesque Metaphysicae," 1678; DM=G. W. Leibniz, "Discourse on Metaphysics," 1686; FW= R. Franks and R. Woolhouse (eds.) G. W. Leibniz: Philosophical Texts, 1998; G=C. I. Gerhardt (ed.) Die Philosophischen Scriften von Gottfried Wilhelm Leibniz, 1978; L=L. Loemker (ed. and trans.), Gottfried Wilhelm Leibniz: Philosophical Papers and Letters $2^{\text {nd }}$ Edition, 1969; LC=Richard Arthur, ed. and trans. G. W. Leibniz, The Labyrinth of the Continuum, Writings on the Continuum Problem, 2001; SD= G. W. Leibniz, "Specimen of Dynamics," 1695; TA=G. W. Leibniz, "Tentamen Anagogicum,” 1696; UO=G. W. Leibniz, "On the Ultimate Origination of Things," 1697. 
geometrical optics from his "Most Determined Path Principle" or "MDPP". The first section places the MDPP in its historical context, and argues that it allows Leibniz to bring to the fore philosophical issues concerning the legitimacy of teleological explanations by addressing two technical objections raised by Cartesians to nonmechanistic derivations of the laws of optics. The second section argues that, by drawing on laws such as the MDPP, Leibniz is able to introduce a thin notion of teleology that gives him the resources to respond to the most pressing charges of his day by showing how teleology within the order of nature may be stripped of problematic Scholastic commitments, fitted to accepted explanatory structures, and successfully applied to a wide and promising range of natural phenomena. Finally, the third section argues that contemporary philosophers have been overly hasty in their dismissal of Leibniz's account of natural teleology, and indeed that their own generally thin conceptions of teleology have left them with few well-motivated resources for resisting Leibniz's elegant position.

\section{Leibniz's Most Determined Path Principle and Its Historical Context}

One of the milestones in the history of optics is marked by Descartes's publication in 1637 of the two central laws of geometrical optics. ${ }^{3}$ The law of reflection - known at least since the time of Euclid - states that the angle at which a ray of light strikes a reflective surface equals the angle at which it is reflected - or, more succinctly, that the angle of incidence is equal to the angle of reflection. The law of refraction - first published by Descartes - states that the ratio between the sine of the angle at which a ray

\footnotetext{
${ }^{3}$ See, especially, the first two discourses of his Optics (AT 81-105/CSM 1:152-164). For an unabridged edition of Descartes's Optics in English, see (Olscamp 1965, 65-173).
} 
of light strikes a refractive surface and the sine of the angle at which it is refracted is a constant determined by the mediums involved.

In keeping with the spirit of his mechanistic program for natural philosophy, Descartes attempts to show how both the laws of reflection and refraction may be derived in an essentially uniform manner by considering broadly mechanical models under idealized conditions. Towards this end, he first argues that the phenomena of light may be modeled on the behavior of moving bodies, insisting that "the action or tendency to move (which, I have said, should be taken for light) must in this respect obey the same laws as motion itself" (AT VI 88/CSM 1:155). He then proposes to derive the law of reflection by considering the behavior of tennis balls being hit by rackets and rebounding off of hard surfaces, and the law of refraction by considering the behavior of tennis balls being hit through thin sheets of cloth (AT VI 93-108). ${ }^{4}$

Descartes's mechanical derivations of the laws of optics received a decidedly mixed response in his own day. On the one hand, the general approach of his project - to explain optical phenomena in broadly mechanical terms - was widely emulated. Inspired largely by Descartes's work, Newton began his famous experiments with prisms around $1665,^{5}$ and his early theory of colors, published less than ten years later, owed much to

\footnotetext{
${ }^{4}$ A more detailed discussion of Descartes's mechanical derivations of the laws of optics is provided in McDonough (2004, 72-77).

${ }^{5}$ John Conduitt reports that "In August 1665, Sir Isaac who was then not twenty-four bought at Sturbridge fair a prism to try some experiments upon Descartes's book of colours and when he came home he made a hole in his shutter and darkened the room and put his prism between that and the wall found instead of a circle the light made [a shape] with straight sides and circular ends ... which convinced him immediately that Descartes
} 
the impetus of Descartes's pioneering project. ${ }^{6}$ Likewise, Huygens's rival wave theory of light, presented to the Académie des Sciences in 1678, grew out of his early acquaintance with Descartes's derivations, and took shape against the background of the general Cartesian framework that he had long since absorbed in his youth (see Sabra 1967, 198-230).

On the other hand, in spite of their undeniable ingenuity, Descartes's particular derivations of the laws of optics met with widespread dissatisfaction. In a letter to an unknown correspondent, Fermat relates that Descartes's account of the laws of optics struck him immediately as "a veritable paralogism" (Fermat 1888-1950, 2: 485). An only slightly moderated sentiment is expressed by Leibniz's complaint that "the way in which Descartes has tried to explain the law of refraction by efficient causes or by the composition of directions in imitation of the reflection of balls is extremely forced and not intelligible enough" (TA 274/L 480). Indeed, many of Descartes's contemporaries took what they saw as the implausibility of Descartes's proofs as evidence that he had, while living in Holland, stolen his results from Willebrord Snell, and tailored his derivations to match the conclusions he had pirated. ${ }^{7}$

was wrong and he then found out his own hypothesis of colours ..." (quoted in Westfall 1986, 157).

${ }^{6}$ See, Newton's letter to Oldenburg reprinted in Newton (1959 1:92-102).

${ }^{7}$ It is clear that Snell had discovered the law of refraction before his death in 1626 . His results, however, were not made public until 1632 when his manuscript was found by Jacobus Golius, and after Descartes had made arrangements to have his own formulation of the law tested empirically. Isaac Vossius was the first to publicly accuse Descartes in 1662 of having learned of the law from Snell's work while he was residing in Holland 
Fermat's own dissatisfaction with Descartes's proofs eventually led him to pursue a radically different approach to the laws of optics. It was already well known in Fermat's day that for standard situations the law of reflection could be derived from the principle that light always takes the shortest reflected path between any two points. ${ }^{8}$ The (Vossius 1662, 36). Huygens, however, had already expressed reservations about the priority of Descartes's discovery (Huygens 1888-1950, 8:9; see also 10:405). Huygens's reservations were clearly shared by Leibniz who remarks in the Tentamen Anagogicum that “... I have no doubt whatever that this law [of refraction] was first discovered by this method [of Snell and Fermat]. It is known that Willebrord Snell, one of the greatest geometricians of his time ... invented it, having even written a work which was not published because of its author's death. But since he had taught it to his disciples, all appearances point to the conclusion that Descartes, who had come to Holland a little later and who was most interested in this problem, learned it there" (TA 274/L 479-80; see also DM 22/L 317-18). For discussion of the originality and priority of Descartes's discovery see especially Sabra (1967, 99-116), who concurs with D. J. Korteweg that “neither the question of Descartes' originality nor that of priority can be settled on the evidence available: he may well have discovered the law of refraction independently of Snell (indeed this is more likely than the contrary supposition), and his discovery may have antedated that of Snell" (Korteweg 1896, 489-501; Sabra 1967, 102-103). The law of refraction is still referred to today as both "Snell's Law" and "The Snell-Descartes Law.”

${ }^{8}$ Heron of Alexandria (ca. A.D. 75) had used essentially the same principle to derive the law of reflection (Herons von Alexandria 1900, IV, 324-9). For discussion see especially Sabra $(1967,71 f)$. 
principle of the shortest path, however, obviously could not be applied directly to cases of refraction, for if a ray of light traveled along the shortest path between two points in different mediums, it should never be bent by the refracting medium, but should rather always travel along a straight line. It was Fermat's insight to realize that although the path of a ray of light could not always be the shortest, it might nonetheless always be the quickest route between two given points. Since in standard cases of reflection, the shortest reflected route coincides with the quickest reflected route, Fermat faced no difficulty in deriving the law of reflection from his new principle. He was also able to easily prove that if light travels faster in one medium than in another, a straight line would not necessarily represent the quickest path since a ray of light might travel more quickly by covering a greater distance in the less resistant medium and a correspondingly shorter distance in the more resistant medium (see, Fermat to La Chambre, 1 January 1662, Fermat 1891-1912, 2:457-63). ${ }^{9}$

Although discovering a general method of directly calculating the shortest path for a refracted ray of light turned out to be mathematically very challenging, Fermat was eventually able to prove - to his own astonishment - that his principle of least time yields essentially the same sine law of refraction as offered by Descartes, which he had originally set out to refute (Fermat 1891-1912, 2:460f). In an at least superficially conciliatory letter, Fermat therefore finally granted that Descartes had published the correct laws of optics, and reserved for himself the claim only to have revealed "the proof

${ }^{9}$ Sabra $(1967,141)$. In the limiting case where an unreflected ray of light remains always in the same medium, it will travel along a straight line, which of course is both the shortest and quickest path (at least under the standard conditions presently being considered). 
of this truth, so important, and which must produce consequences so admirable" (Fermat 1891-1912, 2:462).

The apparent possibility of deriving the laws of optics from two radically different approaches should have immediately raised a host of intriguing methodological and philosophical issues. What sense might be made of Fermat's obviously non-mechanical derivations of the laws of optics? How might they be reconciled with the broadly mechanical approach favored by Cartesians, or, for that matter, with the general metaphysical and explanatory framework of the new science? Investigation into such pressing philosophical questions, however, was stalled by two technical issues that still separated Fermat and his Cartesian adversaries.

The first issue arises most saliently in cases of reflection off of concave surfaces. It had long been know that in certain non-standard cases, a ray of light might take the longest, rather than the shortest, reflected path between its source and sink. Recognizing the challenge to his own quickest path principle, Fermat proposes introducing "another indisputable principle that everything that depends or which is made determinate on a curved line is of such a nature that it is supposed to depend on or be made determinate by a straight line which touches the curve at the point where they touch" (Fermat 1891-1912, 2:354-55). By appealing to tangent planes to reflective surfaces, Fermat is indeed able to make his principle yield the correct results even in non-standard cases of reflection. To his Cartesian adversaries, however, such appeals could only appear as ad hoc attempts to fix a defective principle. ${ }^{10}$ They thus continued to view cases of reflection off of concave

\footnotetext{
${ }^{10}$ Notably, Leibniz was himself much more sympathetic to Fermat's further assumption concerning the appeal to tangent planes (TA 273/L 479 and TA 277/L 483). For
} 
surfaces as providing a clear source of counterexamples to Fermat's quickest path principle.

Second, in deriving the sine law of refraction, Fermat assumes that light travels faster in rarer materials - so, for example, that it travels faster in air than in water. That assumption, however, contradicts the Cartesian view that light travels faster in denser materials. Although seemingly counter-intuitive - real tennis balls, of course, slow down in moving from, say, air to water - Cartesians believed that their position could be conclusively defended on broadly mechanistic grounds. Thus Descartes, for example, after reminding his readers that light is "nothing but a certain movement or an action received in a very subtle matter which fills the pores of other bodies," insists that "the action of this subtle matter can be impeded much more by the parts of the air (which, being as it were soft and badly joined, do not offer it much resistance) than by those of water, which offer it more resistance; and still more by those of water than by those of glass or crystal" (AT VI 103/CSM 1:163). The point of contention here is not merely academic. For, as Clerselier himself notes in response to Fermat's semi-conciliatory letter, the issue of the speed of light relative to its medium is a crucial one for the applicability of Fermat's principle to cases of refraction (Fermat 1891-1912, 2:470). If light were to travel faster in air than in water, as the Cartesians maintained, then it would not generally take the quickest path even in standard cases of refraction. ${ }^{11}$

discussion of some of the broader implications that this commitment has for Leibniz's philosophy of physics, see Bernstein (1984) and Stein (1977).

${ }^{11}$ The question as to whether light travels faster in water or air was not settled experimentally until the mid-nineteenth century when Fizeau (1849) and Foucault (1862) 
Leibniz's most significant contributions to the development of optics all arise out of his attempts to reconcile the two approaches to optics represented by the work of Descartes and Fermat. In attempting to improve upon Descartes's broadly mechanical derivations, Leibniz characteristically emphasizes considerations of continuity and elasticity. ${ }^{12}$ In order to improve upon Fermat's patently non-mechanical approach, Leibniz attempts to address the two technical objections raised by Cartesians to the great mathematician's derivations.

At the center of Leibniz's efforts to improve upon Fermat's derivations lies his "Most Determined Path Principle" or "MDPP". He introduces it as "another principle which supersedes" the principle that "the greatest ease be obtained in relation to planes" insisting that "it remains always universally true that a ray is directed in the most determined or unique path, even in relation to curves" (TA 274/L 479). Put simply, Leibniz's principle is tantamount to the claim that from among all the possible paths between a source and a sink, a ray of light will travel along the path which is unique with respect to ease; where "ease" is understood as the quantity obtained by multiplying the distance of the path by the resistance of the medium(s).

Using his infinitesimal calculus, and the MDPP, Leibniz is able to offer new derivations of the now familiar laws of reflection and refraction. In his most sophisticated treatment - that of the Tentamen Anagogicum - Leibniz begins by first illustrating the use of his calculus to find local maxima and minima, asking his readers to

proved that light does indeed travel faster in air as Fermat had predicted (Ronchi 1970, 259).

${ }^{12}$ See, for example, DC 1404/LC $253 f$. 
consider "a curve $\mathrm{AB}$, concave or convex, and an axis ST to which the ordinates of the curve are referred:"13

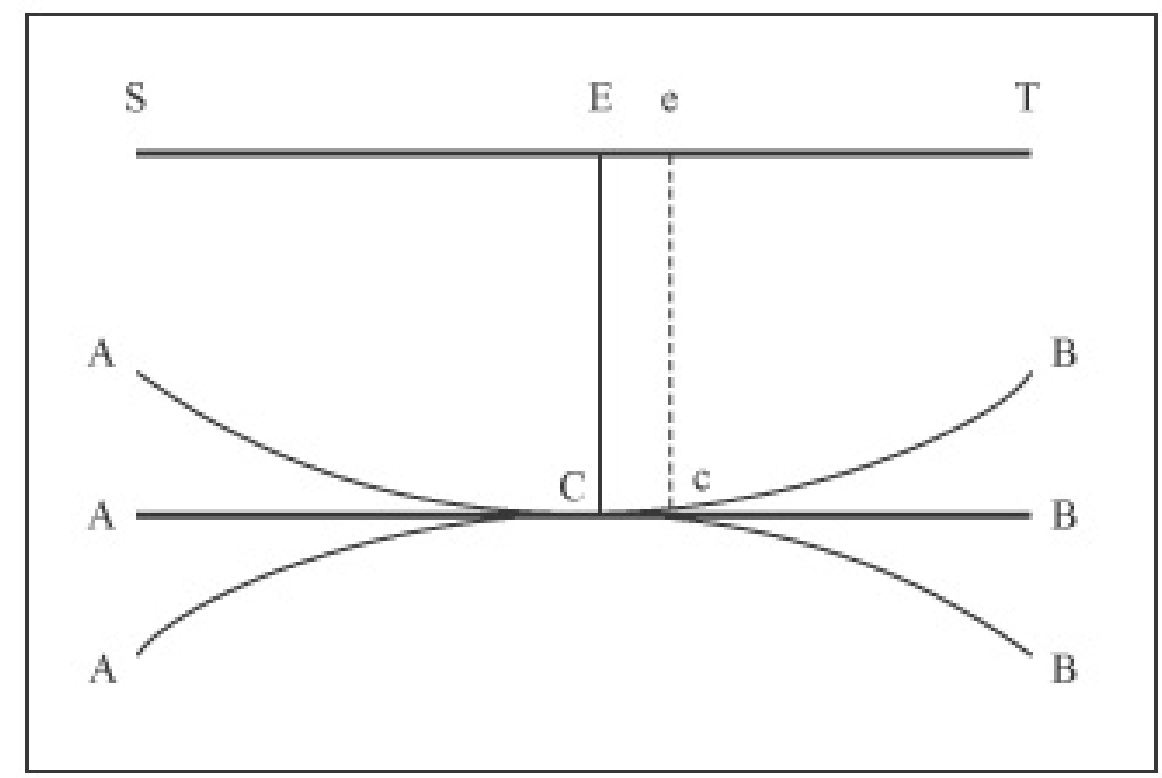

\section{Figure 4}

Leibniz somewhat confusingly states the problem of finding the point $\mathrm{C}$, which is unique with respect to its ordinate (i.e. y-axis) value, in terms of the "union of twins." The intuitive idea behind Leibniz's terminology, however, is simply that $\mathrm{C}$ is the only point on $\mathrm{AB}$ which does not have a corresponding point of the same ordinate value a finite distance away, i.e. C is the only point on AB whose "twin" with respect to ST would have to be "infinitely close." Put in contemporary terms, C is therefore the only point on $\mathrm{AB}$ where the derivative of the line with respect to ST equals zero. As an immediate consequence, given the equation of the line $\mathrm{AB}$, one can therefore find the point $\mathrm{C}$ by taking the derivative of the equation and setting it equal to zero. With good justification,

\footnotetext{
${ }^{13}$ This is a simplified version of Leibniz's own figure 1 (TA 275/L 480). Those interested in Leibniz's derivations of the laws of optics, should also see his (1682).
} 
Leibniz insists that this now standard operation for determining local maxima and minima greatly simplifies the calculations used in his derivations of the laws of reflection and refraction. ${ }^{14}$

In the Tentamen Anagogicum, Leibniz next shows how the law of reflection can be derived from the MDPP with the aid of his calculus. He asks his readers to consider a ray of light traveling between the fixed points $\mathrm{F}$ and $\mathrm{G}$ via a mirror ACB which could be

\footnotetext{
${ }^{14}$ For some sense of how much Leibniz's calculus facilitates his proof, it may be worth noting that it took Fermat more than four years to see how to apply his own method to the problem of refraction (Sabra 1967, 145). See also Fermat's, "Analysis ad Refractiones" (Fermat 1891-1912, 1:170-72); in French translation (Fermat 1891-1912, 3: 149-51).
} 
plane, concave, or convex: $:^{15}$

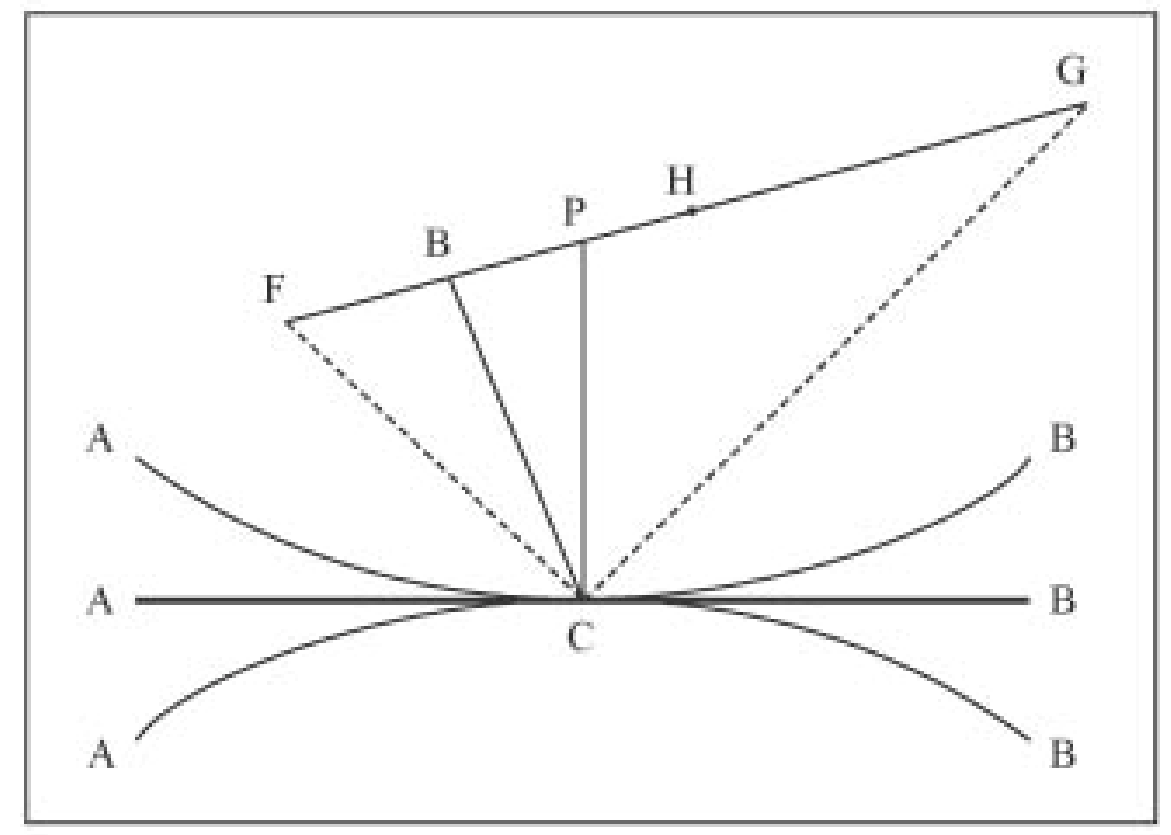

Figure 5

Tacitly assuming that the medium through which the light travels is homogenous and isotropic, Leibniz reduces the problem of finding the unique path with respect to distance times resistance to the problem of finding the point $C$ such that the path FCG is unique with respect to its length. He then (i) constructs an equation for the length of the path from $\mathrm{F}$ to $\mathrm{G}$ via some point $\mathrm{C}$ on $\mathrm{ACB}$, (ii) uses the technique previously illustrated to

${ }^{15}$ This is a simplified, and slightly modified, version of Leibniz's own "figure 1" (TA $275 /$ L 480). It should be noted that a potentially very confusing typo occurs in Loemker's translation. The sentence that appears as "Let ACB be any mirror whatever, plane, concave, or convex; and let two points F and G be given (Figure 34)" should read "Let ACB be any mirror whatever, plane, concave, or convex; and let two points F and G be given (Figure 33)" (L 481). 
find the value of the equation of the path such that the value is unique or "stationary," and then (iii) uses elementary trigonometry to show that for such a path the angle of incidence FCA must be equal to the angle of reflection GCB. ${ }^{16}$

Leibniz uses essentially the same strategy in proving the law of refraction. He asks his readers to consider a refracting surface $\mathrm{ACB}$ which, again, could be planar, concave, or convex, and to let $\mathrm{F}$ and $\mathrm{G}$ represent source and sink points for a ray of light (so that the refracted ray of light will be represented by GCF): ${ }^{17}$

${ }^{16}$ More precisely, Leibniz lets, $\mathrm{HF}=\mathrm{HG}=\mathrm{a}, \mathrm{HB}=\mathrm{x}, \mathrm{CB}=\mathrm{y}, \mathrm{CB} \perp \mathrm{FG}, \mathrm{CP} \perp \mathrm{ACB}$. Since $\mathrm{CBP}$ is a right triangle, $d y=\mathrm{PB} / \mathrm{CB}$. Substituting $\mathrm{y}$ for $\mathrm{CB}, \mathrm{dy}=\mathrm{PB} / \mathrm{y}$. Multiplying through by y and taking the distance from B to $\mathrm{P}$ to be negative, $-\mathrm{ydy}=\mathrm{BP}$. Now since $\mathrm{CBF}$ is also a right triangle, $\mathrm{CF}=\sqrt{ }\left((\mathrm{CB})^{2}+(\mathrm{BF})^{2}\right)$. Substituting y for $\mathrm{CB}$ yields $C F=\sqrt{ }\left(y^{2}+(B F)^{2}\right)$. But $B F=a-x$, so by substitution, $C F=\sqrt{ }\left(y^{2}+(a-x)^{2}\right)=\sqrt{ }\left(y^{2}\right.$ $\left.-2 a x+a^{2}+x^{2}\right)$. Similar considerations show that $C G=\sqrt{ }\left(y^{2}+(a+x)^{2}\right)=\sqrt{ }\left(y^{2}+2 a x+a^{2}\right.$ $+x^{2}$ ). In order to find the path $F C G=C F+C G$ which is unique with respect to length, Leibniz differentiates and sets the resulting equation equal to zero: $d \cdot C F+d \cdot C G=d$ • $\sqrt{ }\left(y^{2}-2 a x+a^{2}+x^{2}\right)+d \cdot \sqrt{ }\left(y^{2}+2 a x+a^{2}+x^{2}\right)=(y d y+x d x-a d x) / C F+(y d y+x d x+$ $\mathrm{adx}) / C G=0$. Rearranging terms yields: $\mathrm{CF} / \mathrm{CG}=(\mathrm{a}-\mathrm{x}-\mathrm{ydy}) \mathrm{dx} /(\mathrm{a}+\mathrm{x}+\mathrm{ydy}) \mathrm{dx}$. Substituting $\mathrm{a}-\mathrm{x}$ for $\mathrm{BF}$, and $\mathrm{a}+\mathrm{x}$ for $\mathrm{GB}$ in turn yields: $\mathrm{CF} / \mathrm{CG}=\mathrm{BF}+\mathrm{BP} / \mathrm{GB}-\mathrm{BP}=$ $\mathrm{PF} / \mathrm{PG}$. Trigonometry is now sufficient to show that if $\mathrm{CF} / \mathrm{CG}=\mathrm{PF} / \mathrm{PG}$, then $\mathrm{CP}$ bisects FCG, and that the angle of incidence is therefore equal to the angle of reflection.

17 “Figure 2" (TA 276)=“Figure 34” (L 481) 


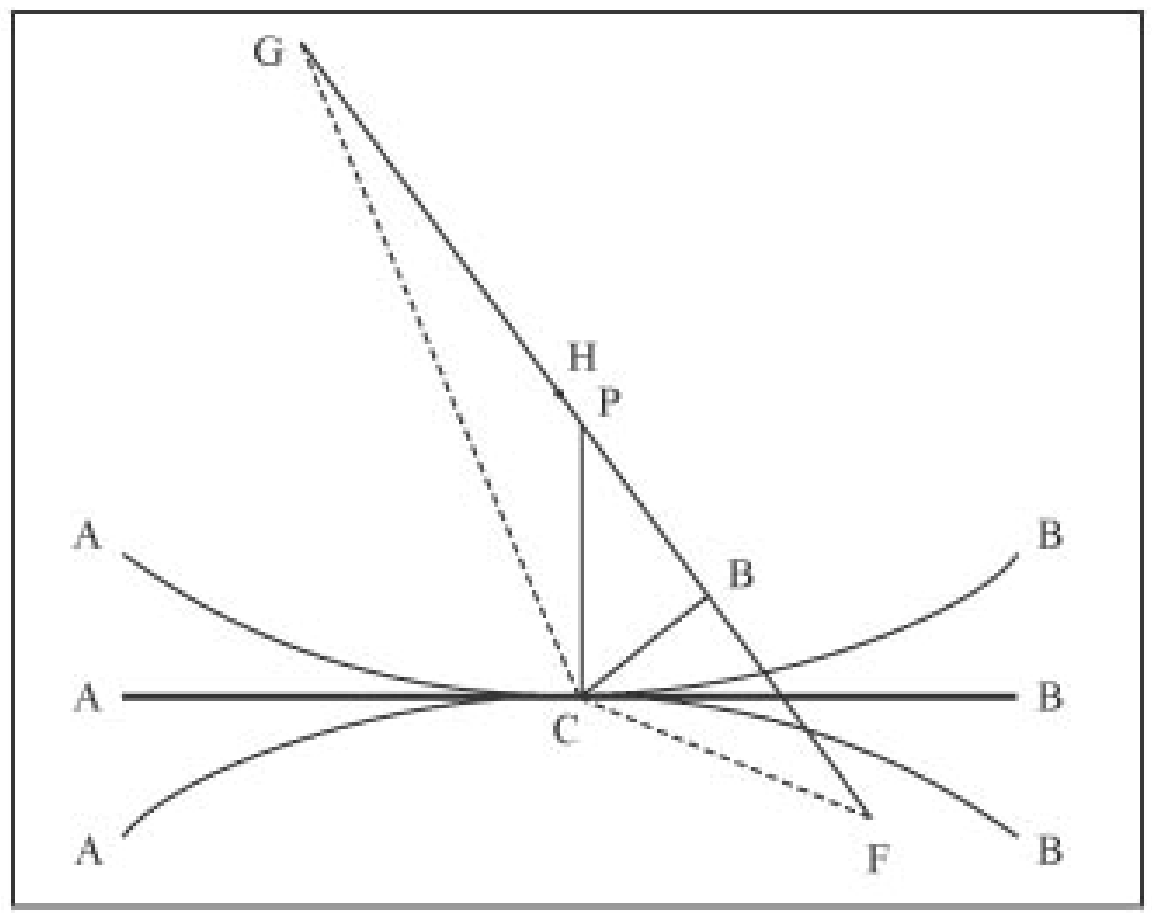

\section{Figure 6}

Here Leibniz once again (i) constructs an equation for the path of the ray of light - this time taking into account the different resistances of the two mediums, (ii) applies his calculus to find the path that is unique with respect to ease (i.e. length times resistance), and (iii) uses trigonometry to show (a) that the ratio of the sine of incidence to the sine of refraction is inversely proportional to the ratio of incident velocity to the refractive velocity, and (b) that the ratio between the sine of the angle at which a ray of light strikes a refractive surface and the sine of the angle at which the ray is refracted is a constant determined by the mediums involved. ${ }^{18}$

${ }^{18}$ In more detail, Leibniz lets $\mathrm{HF}=\mathrm{HG}=\mathrm{a}, \mathrm{HB}=\mathrm{x}, \mathrm{CB}=\mathrm{y}, \mathrm{CB} \perp \mathrm{FG}, \mathrm{CP} \perp \mathrm{ACB}$ As before, $F C G=C F+C G=\sqrt{ }\left(y^{2}+(a-x)^{2}\right)+\sqrt{ }\left(y^{2}+(a+x)^{2}\right)=\sqrt{ }\left(y^{2}-2 a x+a^{2}+x^{2}\right)+$ $\sqrt{ }\left(y^{2}+2 a x+a^{2}+x^{2}\right)$. Taking the resistance of the upper medium to the lower medium to be as $\mathrm{f}$ to $\mathrm{g}$, then the measure of the ease of the path $\mathrm{FCG}=\mathrm{f} \cdot \mathrm{CF}+\mathrm{g} \cdot \mathrm{CG}=\mathrm{f} \cdot \sqrt{ }\left(\mathrm{y}^{2}-\right.$ 
It is perhaps worth noting explicitly here that Leibniz's derivations of the laws of optics illustrate - in a somewhat elementary fashion - the central idea of what has become know as the method of variations. Leibniz's equations, in effect, allow him to represent a family of possible paths that a ray of light might take between its source and its sink. Using his calculus, he is then able to identify a uniquely distinguishing property of the actual path, namely, that it makes extremal - i.e. locally maximal or minimal - the quantity of "ease." He is then able to pick out, as it were, the one actual path taken by a ray of light from the infinitely many possible paths - or "variations" - described by his initial equations. Although the modern "calculus of variations" generally requires a higher degree of mathematical sophistication (in part because it typically represents the class of "variations" in terms of functionals) it is nonetheless essentially a development of the fundamental strategy already on display in Leibniz's optical derivations. ${ }^{19}$

Armed with his Most Determined Path Principle, and his novel derivations of the laws of optics, Leibniz is able to address both of the technical objections raised by Cartesians against Fermat's proofs. Unlike Fermat's principle, Leibniz's principle requires no special assumptions in order to be applied to non-standard cases of reflection.

$\left.2 a x+a^{2}+x^{2}\right)+g \cdot \sqrt{ }\left(y^{2}+2 a x+a^{2}+x^{2}\right)$. In order to find the path $F C G=C F+C G$ which is unique with respect to its ease (i.e. distance times length), Leibniz once again differentiates and sets the resulting equation equal to zero: $\mathrm{f}(\mathrm{d} \bullet \mathrm{CF})+\mathrm{g}(\mathrm{d} \bullet \mathrm{CG})=\mathrm{f}(\mathrm{d} \bullet$ $\left.\sqrt{ }\left(y^{2}-2 a x+a^{2}+x^{2}\right)\right)+g\left(d \cdot \sqrt{ }\left(y^{2}+2 a x+a^{2}+x^{2}\right)\right)=f \cdot(y d y+x d x-a d x) / C F+g \bullet$ $(\mathrm{ydy}+\mathrm{xdx}+\mathrm{adx}) / \mathrm{CG}=0$. Calculating as above, yields: $\mathrm{CF} / \mathrm{CG}=(\mathrm{f} \bullet \mathrm{PF}) /(\mathrm{g} \bullet \mathrm{PG})$. Trigonometry is now sufficient to complete the proof.

${ }^{19}$ For more on the history of the development of the calculus of variations, see Goldstine (1980), Mach (1989), and Woodhouse (1810). 
For Leibniz, the characteristic feature of actual paths of rays of light is not that they always minimize some quantity, but rather that they are (locally) unique with respect to some quantity. As a result, the possibility that a ray of light might maximize distance, speed, or ease does not present even a prima facie difficulty for his treatment, and he thus has no need to appeal to planes tangent to reflecting surfaces in order to handle nonstandard cases of reflection.

By selecting "ease" as the quantity that is made locally determinate by the actual paths of rays of light, Leibniz is also able to address the objection raised by Cartesians concerning the speed of light in refractive mediums. Although it is natural to see that hypothesis today as rather counterintuitive, in Leibniz's day, it was, in fact, widely thought to be essential to a corpuscular analysis of the behavior of light. It is therefore crucial to Leibniz's reconciliation project that his principle allows him to side with the Cartesians in maintaining that light travels faster in denser materials, while still insisting that light always takes the most determinate path with respect to ease. In this way, Leibniz again effectively removes a crucial barrier separating proponents of the Cartesian and Fermatian approaches to the laws of optics.

Through his novel derivations, Leibniz was thus able to remove the most important obstacles separating the Cartesian and Fermatian approaches to the laws of optics. In doing so, he was thereby able to shift the focus of discussion from technical matters to the more philosophical issues surrounding the legitimacy of non-mechanical explanations in the natural sciences, and their relationship to more familiar explanatory models. He was, in short, able to raise once again the question of the legitimacy of teleological explanations and their compatibility with the "mechanistic" explanations more generally favored by proponents of the new science. 


\section{The Most Determined Path Principle and Natural Teleology}

While the MDPP clearly represents an important contribution to the development of early modern optics, its greatest significance for Leibniz is to be found in his defense of teleological explanations. Throughout his mature period, Leibniz repeatedly emphasizes that principles such as the MDPP provide new grounds not only for linking God's benevolent intentions to the laws of nature, but also for recognizing the utility of non-mechanistic explanations cast in terms of final causes:

In fact (as I have shown by a quite remarkable example of a principle in optics, which the famous Molyneux greatly approved of in his Dioptrics), final causes can sometimes also be introduced to great effect in particular problems in physics - not only so that we can better admire the most beautiful works of the supreme Creator, but also sometimes in order to find out things which by consideration only of efficient causes would be less obvious, or only hypothetical. (SD 24/FW 164)

For I believe that God considered principles of wisdom and reasons of order when he established the laws which are observed in nature. And I think that this makes it clear (as I once pointed out when discussing the laws of optics, and which the famous Molyneux later accepted in his Dioptrics) that consideration of final causes is useful not only to virtue and piety - in ethics and natural theology - but also for discovering and detecting hidden truths in physics itself. (NI 4/FW 212) 
What is more, our thinking sometimes furnishes us with considerations revealing the value of final causes, not merely in increasing our admiration for the supreme Author, but also in making discoveries among his works. Some day I shall show this in a special case in which I shall propose as a general principle of optics ... (TA 273/L 479)

As these passages suggest, principles like the MDPP enter into Leibniz's general defense of teleology in two very different ways. ${ }^{20}$ First, he maintains that, like the laws of motion, principles such as the MDPP can be related to God's intentions to create the best of all possible worlds. In this way, he sees his work in optics as being related to his defense of divine teleology - an important topic for understanding Leibniz's mature philosophy of physics, but one that will not be pursued further in this essay. ${ }^{21}$ Second, Leibniz maintains that principles such as the MDPP can be used to show that teleological explanations have an important role to play even in the thick of doing physics. In order

${ }^{20}$ A contemporary reader might be surprised that Leibniz should place such emphasis on, and try to draw far reaching conclusion from, his work in optics, an area that might seem today to be a rather narrow and specialized sub-field of physics. For that reason, it is perhaps worth recalling that no one in Leibniz's time doubted that optics was one of the central sciences and deserved to share the scientific stage with astronomy and mechanics. It is no accident, for example, that Descartes's first systematic account of nature was titled The World or Treatise on Light, and that Newton was as much known in his own day for his Optics as he was for the Principia.

${ }^{21}$ This topic is discussed in McDonough (2007) and (2004, 113-140). 
to better appreciate Leibniz's suggestion that the laws of optics lend support to a kind of natural teleology, or teleology within the order of nature, it will be helpful to first take a closer look at what Leibniz sees as a contrast between efficient and teleological laws of nature.

As part of the general metaphysical and explanatory framework of the new science, mechanistic philosophers had long countenanced explanations in terms of general efficient laws that straightforwardly link prior events to later events. So, for example, it was widely thought that the laws of motion should relate the speeds and directions of a pair of bodies before a collision to their speeds and directions after the collision. As part of this picture, it was, of course, also accepted that by citing the initial speeds and directions of the bodies together with the laws of motion, one could explain the speeds and directions of the bodies after the collision.

For Leibniz this widely accepted model of explanation also holds out the possibility of a kind of teleological explanation. Leibniz suggests that in addition to seeing the world as being governed by efficient mechanical laws, it might also be viewed as being governed by teleological laws such as the MDPP. Such laws would be teleological in that they would link prior events to subsequent events by appealing to consequences of those subsequent events. Leibniz suggests that if an event could be deduced from a teleological law together with initial conditions and outcome conditions, then one would have a teleological explanation of that event.

In order to see more clearly how Leibniz's distinction between efficient and teleological laws of nature grounds his position on natural teleology, it might be helpful to consider two alternative explanations for a ray of light's passing through a particular 
point of reflection, $\mathrm{B}$, in a simple case of reflection (as depicted in the following diagram):

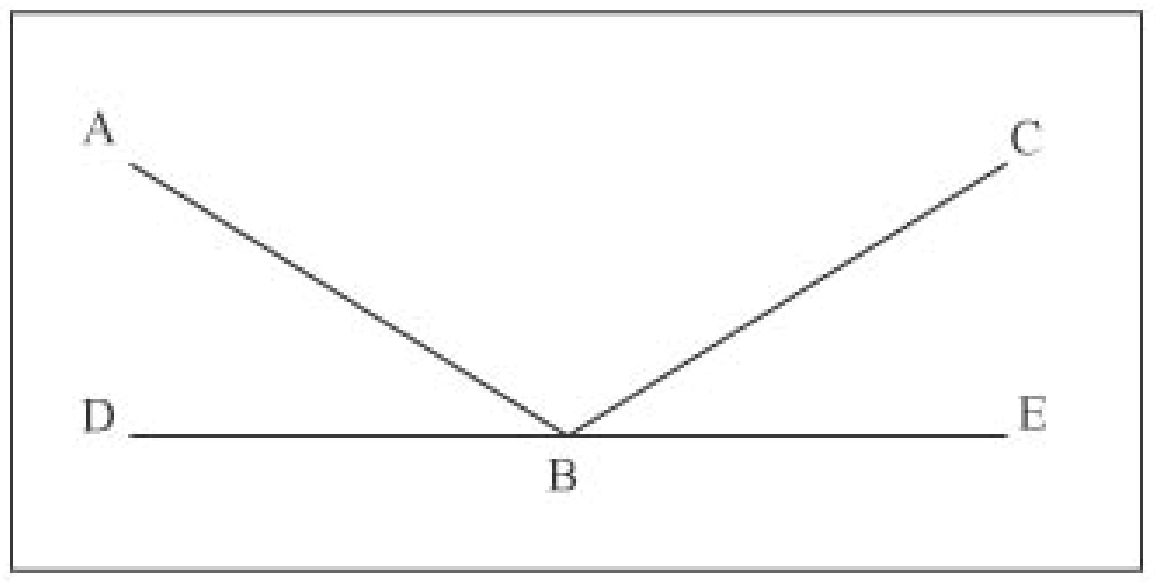

\section{Figure 7}

A typical efficient explanation of the sort offered by Descartes would explain the ray of light's passing through the point B by appealing to its initial speed and direction at point A, and using the laws of motion to derive its position at the next instant. In this way, Cartesians would, in effect, trace the progress of the light infinitesimal piece by infinitesimal piece from point $A$ through to point $B$, and then onto point $C$. In spite of his dissatisfaction with Descartes's actual proofs, Leibniz is confident - perhaps overly confident - that some such efficient explanation of the behavior of rays of light is always at least in principle possible.

In addition to such efficient explanations, however, Leibniz suggests that there is also available a more readily accessible teleological explanation of the same event. Leibniz takes as given that the ray starts at point $\mathrm{A}$ and ends at point $\mathrm{C}$. He then uses his Most Determined Path Principle - which in this case is equivalent to the claim that the ray of light will take the easiest reflected path - to deduce that the ray passes through 
point B. In this example, "reaching its endpoint by the easiest path" serves as the goal of the process by which the ray passes through point $\mathrm{B}$, and we could say in common terms that the ray passes through $\mathrm{B}$ in order to get to $\mathrm{C}$ by the easiest path. ${ }^{22}$

In Leibniz's day no one seems to have doubted that explanations such as those involving the MDPP involve final causes, and thus Leibniz never bothers to spell out explicitly why he thinks they are teleological. Nonetheless it is not hard to see, even in the simple case just presented, at least two prominent features that must have lent considerable support to the consensus. The first such feature - almost too obvious to mention - is that Leibniz's explanation makes essential appeal to an outcome or "goal" of the process by which the ray of light comes to pass through point B. In this way it is similar to other commonly accepted teleological explanations such as that the bear is

${ }^{22}$ The description of the goal of the ray as "reaching its endpoint by the easiest path" might initially strike one as odd. Shouldn't the goal simply be "reaching its endpoint" or "reaching point C"? If the stated goal seems long-winded, it is because it fills in some detail that might reasonably be taken for granted in many contexts. "Reaching its endpoint" would do, but only if it is assumed that it reaches its endpoint by an efficient route, which, of course, is commonly assumed and might therefore go without saying, as when we explain that the heat-seeking missile turned right in order to hit its target (and thus take for granted that it doesn't, say, take three lefts, or circle the globe). "Reaching point C" would likewise do, but only if we assume that some features of the set-up are constant, for example, that the mirror isn't rotated, and the medium remains isotropic. But, again, such features are often assumed to be constant, and therefore again might go without saying, as they typically do, for example, in problem sets commonly assigned to undergraduates. 
swatting at the beehive in order to get honey, which purports to explain the bear's earlier behavior of swatting at the beehive in terms of the result or goal of its swatting at the beehive, namely, its getting the honey. ${ }^{23}$

The second feature of Leibniz's explanation that must have supported its characterization as teleological is that the behavior of the ray of light is shown to be responsive to changes in its environment in exactly the sorts of ways necessary to bring about the goal specified in the explanation. If the ray of light had started at a different origin, passed through a different medium, or been reflected off of a differently configured mirror, it would have behaved differently but still in such a way as to take the easiest path between it source and sink. In this, Leibniz's explanation is again similar to other commonly accepted teleological explanations. Part of what makes the explanation of the bear's behavior convincingly teleological, for example, is that under different circumstances, the bear would have behaved differently - perhaps going around a barrier, or climbing a tree - but still in such a way as to obtain the honey. ${ }^{24}$

${ }^{23}$ Should it be objected that the two explanations offered here differ in the fact that while rays of light always reach their endpoints by easiest paths, bears (for example) do not always succeed in obtaining honey by the means available to them? I think doing so would be misguided, for surely it is not part of our concept of a teleological explanation that it must sometimes fail. Certainly, when theists appeal to divine ends, there is no assumption that God's teleology must be fallible, and if, for example, we built guidedmissiles that never missed their targets, their reliability would hardly seem to undermine teleological explanations of their behavior.

${ }^{24}$ Conversely, we would not normally say that the bear is swatting at the beehive in order to get stung even if that is a consequence of his swatting at the beehive precisely because 
While Leibniz's characterization of his proposed explanations as teleological was thus embraced by his contemporaries - who typically objected to them precisely because they agreed that they are teleological - it is worth noting that the notion of teleology at play in Leibniz's explanations is significantly thinner than those of his Aristotelian predecessors in at least two important respects. First, it was one of the hallmarks of Aristotle's position on teleology that final causes are metaphysically immanent in nature - that they are the immediate result of robust formal natures that are intrinsically directed towards their own appropriate ends. ${ }^{25}$ To be sure, such teleological natures are not entirely foreign to Leibniz's mature metaphysics, and simulacra of them are found in his construal of monads as primitive entelechies, and play an important role in his rejection of occasionalism. ${ }^{26}$ Nonetheless, the final causes that arise in connection with laws such he would presumably not have modified his behavior in order to reliably bring about his getting stung. This consideration highlights an important difference between the kinds of explanations Leibniz is defending, and "explanations" that would try to take advantage of the temporal "reversibility" of the laws of nature in order to deduce prior states of a process from later states of that processes. Although one could deduce the velocity and position of a billiard ball at $t_{1}$ from its velocity and position at $t_{2}$, we would not normally deem this to amount to a teleological explanation at least in part because we do not think that the billiard ball would have adjusted it behavior in order to arrive at that position, with that velocity, at $t_{2}$. These sorts of considerations will resurface more prominently in the third section.

${ }^{25}$ On this point, see, especially, Hull $(1981,282)$.

${ }^{26}$ For further discussion of the connection between Leibniz's postulation of active natures and his rejection of occasionalism, see, Rutherford (1993). 
as the MDPP, and that, according to Leibniz, are of concern to the practicing physicist, are grounded immediately only in the structure of the laws of nature. As a consequence, Leibniz's conception of teleology is not directly tied to any metaphysical picture of goaldirected strivings, and is at least consistent with any view - including occasionalism and "Humeanism" - that attributes law-like regularities to the natural world.

Second, in keeping with his primary focus on the biological realm, Aristotle suggests that genuine teleology must not only reliably bring about a given end state, but that the end state in question must be related to the good of the relevant substance. ${ }^{27}$ For Aristotle, it is therefore not enough for genuine teleology that a tree should consistently act in such a way as to develop leaves, but rather it must develop leaves for the sake of protecting its fruit, and thus for the sake of contributing to its own flourishing. On Leibniz's view, however, it is not necessary to suppose that rays of light take optimal paths for the sake of their own good; it is sufficient that they reliably act in such a way as to consistently bring about a particular end state. Considerations of goodness enter only at one step removed, when the contribution of rays of light to the overall perfection of the universe is brought into the picture. In this respect, Leibniz's position is something of an intermediary between Aristotle's - where considerations of the good must play an immediate role - and the accounts of contemporary philosophers, which typically attempt to divorce altogether evaluative judgments from teleological attributions. ${ }^{28}$

If Leibniz's contemporaries were happy to agree that his proposed explanations are teleological, they were less convinced about the legitimacy of those explanations. Much of their resistance stemmed from the association of teleological explanations with

${ }^{27}$ On this point, see, especially, Cooper (1987).

${ }^{28}$ For important dissent from this general trend, see Bedau (1992a and 1992b). 
Scholasticism. As part of the metaphysical and explanatory framework of Scholastic natural philosophy, final causes typically entered into their scientific accounts of natural phenomena in two importantly different ways. First, Scholastic philosophers generally analyzed all natural changes as being inherently directed towards specific goals as determined by their relevant forms. In virtue of their elemental forms, heavy bodies, for example, were taken to be naturally directed towards their final resting place at the center of the universe, while in virtue of their substantial forms, acorns and fawns, for example, were taken to be naturally directed towards their mature development as oak trees and deer. Insofar as teleological explanations of Scholastic natural philosophers relied upon such irreducible, qualitative, forms, they were seen as being of a piece with what mechanical philosophers viewed as the bankrupt explanatory and metaphysical framework of Scholastic natural philosophy more generally.

Second, Scholastic philosophers not infrequently made bald appeals to God's ends in attempting to explain particular natural phenomena. A striking example of teleology used in this way is provided by Abra de Reconis's account of the saltiness of the sea, which he explains in terms of "the two most important ends to which the sea is instituted," namely, "first, that it should be the common domicile of fish, and second that in it there should be navigation to provide commerce and necessary goods" both of which are promoted by salinity "since saltiness keeps the sea from putrefying and makes it stronger and denser so as to hold the greater weight of ships." ${ }^{29}$ For many seventeenth century mechanical philosophers, such teleological explanations not only smacked of the

${ }^{29}$ I borrow the example, as well as the translation, from Des Chene $(1996,168)$. The original text may be found in Abra de Raconis $(1651,384)$. 
idle, and non-confirmable, speculations that plagued Scholastic philosophy in general, but also involved presumptuous speculation concerning God's ends. ${ }^{30}$

Leibniz is clearly sympathetic with the reservations of mechanist philosophers concerning Scholastic attempts to introduce final causes into scientific explanations. Although he defends the existence of immaterial entelechies as part of his fundamental metaphysics, he nonetheless denies that such formal natures should be appealed to in scientific explanations of particular natural phenomena. Thus in the Specimen Dynamicum, for example, he writes:

Meanwhile, although I hold that there exists everywhere in bodies an active or, as we might say, vital principle which stands above all material concepts, I do not agree with Henry More and other men of outstanding piety and intelligence who appeal to some kind of unheard of archeus or hylarchic principle in explaining the phenomena - as if not everything can be explained mechanically, and as if those who attempt to give such an explanation were to be suspected of impiety for trying to deny incorporeal things; or as if we ought to assign intelligences that were required to rotate the spheres, as Aristotle did, and say that the rising and falling of the elements is due to their forms - a theory which covers a great deal, but which tells us nothing. (SD 22/FW 163; see also SD 6/FW 156, and DM 10/FW 61)

Likewise, although Leibniz grants that every physical event must ultimately be determined by God's ends in creating the best of all possible worlds, he nonetheless insists that in physics, God's direct influence should only be appealed to in explanations

${ }^{30}$ See Descartes's Principles 1:28, and Bacon (1858, 1:151). 
of general principles and not of particular phenomena. ${ }^{31}$ He thus maintains that "it is empty to resort to the first substance, or God, in explaining the phenomena of his creatures, unless his means or ends are, at the same time, explained in detail, and the proximate efficient or even the pertinent final causes are correctly assigned, so that he shows himself through his power and wisdom" (G IV 397-398/AG 254).

Beyond distancing himself from what were seen as Scholastic excesses, Leibniz is also able to use his optical explanations to address two specific mechanistic objections to the use of teleological explanations in natural philosophy. The first of these objections is that the postulation of final causes within the order of nature commits one to attributing thought or intentionality to unintelligent entities. ${ }^{32}$ In a neglected discussion of reflection, Leibniz remarks that

[C]ertain people of more recent times have been wont to object that the ray coming from A is not endowed with cognition, nor does it inquire whether it will go to $\mathrm{C}$ and which is the best rout for it to get there, but with blind impetus comes down on a point on the reflecting surface, to which it is carried in the direction already conceived, and from there rebounds according to mechanical laws. (DC $1405 /$ LC 255f)

As Leibniz goes on to note, however, this objection fails to appreciate the nature of the teleological explanations being offered. According to Leibniz, the path of a ray of light is determined not by any decision or foresight on the part of the ray, but rather because its

${ }^{31}$ See, for example, SD 23/FW 163.

${ }^{32}$ Cf. Nagel $(1961,402)$ : “because of the association of teleological explanations with the doctrine that goals or ends of activity are dynamic agents in their own realization, it [i.e. modern physics] tends to view such explanations as a species of obscurantism." 
behavior is controlled by a law of nature from which it follows that the ray will pass through some point B such that its total path will be optimal. Such laws no more require thought on the part of unintelligent entities than do mechanical laws governing the behavior of material bodies. Indeed, if foresight is required here at all, it will be the foresight of God who is responsible, according to Leibniz, for all the laws of nature, mechanical and teleological alike.

The second specific objection common among mechanists to the use of teleological explanations in natural philosophy is that such explanations must introduce explanatory gaps in the network of non-teleological, efficient, causal explanations, and thus undermine the universal applicability of mechanical explanations. Against this criticism, Leibniz insists that the way of final causes in no way undercuts the completeness of the way of efficient causes. Indeed, he shrewdly recognizes that justifying teleological explanations by the failure of mechanical explanations leaves the defender of teleology unnecessarily open to easy refutation:

We know that ... learned and zealous theologians who, shocked at the corpuscular philosophy and not content with checking its misuse, have felt obliged to maintain that there are phenomena in nature which cannot be explained by mechanical principles; as for example, light, weight, and elastic force. But since they do not reason with exactness in this matter, and it is easy for the corpuscular philosophers to reply to them, they injure religion in trying to render it a service, for they merely confirm those in their error who recognize only material principles. (TA 272/L 478; see also DC 1403/253, and G III 607/L 655) Leibniz, in fact, accepts the dogma of his day that "all natural phenomena could be explained mechanically if we understood them well enough" (TA 272/L 478), and even 
insists that explanations in terms of efficient causes are "deeper and in some way more immediate and a priori” (DM 22/FW 74). Nonetheless, he also sensibly maintains both that the existence of non-teleological explanations in no way rules out the possibility of teleological explanations (properly understood), or vice versa, and that if justification for the postulation of teleological laws is required it can be had by the fact that "the way of final causes is easier, but is nevertheless frequently of use in discovering important and useful truths, truths which one would take a long time to find by the other, more physical route" (DM 22/FW 74).

Although Leibniz thus makes a strong case for the compatibility of teleological laws and mechanism, his boast that the way of final causes is "frequently of use in discovering important and useful truths" points towards what was no doubt the de facto greatest obstacle to his proposed revival of teleological explanations within physics, namely, the need to discover a wide and promising range of natural phenomena that could be explained by teleological laws similar to the MDPP. While it must be admitted that the laws of optics occupy a privileged place in seventeenth century physics - with their investigation playing a prominent role in the work of Descartes, Huygens and Newton among others - Leibniz's ambitious proposal might well appear rash if it were based solely on the basis of the MDPP. ${ }^{33}$ It is therefore significant that - although Leibniz tirelessly draws attention to the teleological significance of his own optical

${ }^{33} \mathrm{Cf}$. Bennett's remark: "Leibniz is not here merely repeating his point that patterns in nature bring in teleology because they reveal God's purposes. He says that asking a teleological question could help one to discover Snell's law. Every time he reiterates that teleology can ('frequently') help one to do physics, he adduces this one example" (Bennett 2001, 1:269). 
explanations - he is also able to point towards at least three other classes of problems that could be solved using essentially the same techniques that he had used in deriving the laws of optics, and which thereby invited similar teleological interpretations.

The first such class of problems involves the maximization of an area or volume for a given perimeter or enclosing surface area. Problems of this kind were already well known to the ancient Greeks, who gave them physical import by suggesting that nature must implicitly solve them in some of its works. So, for example, it was claimed that the hexagonal structure of honeycomb could be explained by the fact that nature attempts to maximize the storage capacity of the cells of the honeycomb while minimizing the amount of wax needed to construct it. In a similar fashion, Leibniz suggests that the spherical shape of a drop of liquid suspended in another liquid can be explained by appealing to "a principle of determination in nature which must be sought by maxima and minima; namely, that a maximum effect should be achieved with a minimum outlay, so to speak" (UO 303-304/L 487-88; see also Leibniz (1697b), 10). (Essentially the same strategy is still employed today in explaining the shape of physical phenomena ranging from soap bubbles to galaxies. ${ }^{34}$ )

In 1690, Leibniz was introduced to a second class of problems by James Bernoulli who proposed as a challenge the determination of the shape of the catenary - that is of a uniform, heavy, flexible, freely hanging cord, e.g. a freely hanging chain suspended at both ends. ${ }^{35}$ Clearly impressed by the significance of Bernoulli's problem, Leibniz

\footnotetext{
${ }^{34}$ For a more general discussion of this first class of problems, see Mach $(1989,479-515)$ as well as Hildebrandt and Tromba (1985).

${ }^{35}$ For a helpful discussion of James Bernoulli's role in stimulating investigation of the problem of the catenary, see Hofmann (1970-1980, 47f). For general discussion of
} 
solved it immediately by drawing upon the resources of his newly developed calculus. The solution he provided, as well as those proposed by Huygens and Johann Bernoulli (James's younger brother), was published in 1691 in the Acta Eruditorum. Subsequent work (especially by the Bernoulli brothers) showed how solutions could be similarly obtained for more complex cases such as those of elastic and non-uniform chains, as well as bent elastic beams. By 1697, Leibniz was comfortable enough with this class of problems to simply assert without argument that "when many heavy bodies pull upon each other, the resulting motion is such that the maximum possible total descent is secured" (UO 304/L 488).

In 1696, Johann Bernoulli introduced Leibniz to yet another class of problems when he first published "the problem of the brachistochrone" in the June edition of the Acta Eruditorum, and then - at Leibniz's request - republished it as a leaflet for quicker distribution. ${ }^{36}$ The challenge it presented to "the most acute mathematicians flourishing in the whole world" was to find the path of quickest descent between two points in the vertical plane for a freely falling body. Again using his infinitetesimal calculus, Leibniz was able to solve the problem on the day he received it, and correctly predicted that only four other solutions would be provided - one from each of the Bernoulli brothers, one from Newton, and one from L'Hopital. Of these four responses, Johann Bernoulli's drew the most explicit connection between the brachistochrone and the laws of optics. Indeed, problems connected with the catenary, see Mach (1989, 86-89) and Lemons (1997, 55$56)$.

${ }^{36}$ For a brief, but interesting discussion of the Bernoulli brothers' work on the problem of the brachistochrone, see Fellmann and Fleckenstein (1970-1980). For a general discussion of the problem, see Mach (1989, 520-26) and Lemons (1997, Chapter 2). 
his brilliant insight was to see that the path of quickest descent could be discovered by treating a falling body as a ray of light traveling through a medium in such a way that it accelerates at the same rate as would a freely falling body. James Bernoulli's solution, while less elegant in some respects, nonetheless highlighted a feature of the problem that would ultimately lead to the development of the calculus of variations proper. His proof emphasized the fact that any portion of a path of quickest descent must itself be a path of quickest descent, and as a consequence such a path can be thought of as the path which is such that any sub-path of it is unique with respect to quickest descent. It is this feature of James Bernoulli's solution that is echoed in Leibniz's remark in the Tentamon Anagogicum that "in these forms or figures the optimum is found not only in the whole but also in each part, and it would not even suffice in the whole without this. For example, if in the case of the curve of shortest descent between two given points, we choose any two points on this curve at will, the part of the line intercepted between them is also necessarily the line of shortest descent with regard to them" (TA 272/L 478).

Leibniz's work on problems such as finding the shape of the catenary and the brachistochrone lie behind his confidence that the techniques he employed in his optical discoveries could be extended to a wide range of phenomena. Given that those techniques were still in their infancy, his conviction was no doubt both optimistic and bold. Nonetheless, it can hardly be denied that Leibniz had a strong hand to play against his early modern opponents: Everyone was willing to concede that his explanations were teleological; he had convincing responses to the standard objections of his day to the use of teleological explanations; and - although it would perhaps not be fully appreciated by his less mathematically sophisticated opponents - he had at his disposal growing evidence that his favored explanations would generalize successfully. 


\section{An Old Myth about Teleology?}

The development of mathematical physics since Leibniz's day would seem to have only strengthened his hand further. In 1747, Maupertuis introduced his famous principle of least action according to which "in all the changes that take place in the universe, the sum of the products of each body multiplied by the distance it moves and by the speed with which it moves is the least possible" (Maupertuis1698-1759, 2:274). Although obscure and incomplete as it stood, the principle of least action was later clarified, developed, and set on secure mathematical foundations through the work of Euler, Lagrange, Hamilton, and Jacobi among others. Its descendants - known today as "variational principles" - have been successfully applied to optical, dynamical, and electromagnetic problems in classical mechanics and continue to play important roles in the context of quantum mechanics and the theories of special and general relativity. ${ }^{37}$

In spite of the remarkable success of variational principles within the natural sciences, however, contemporary philosophers have been reluctant to follow Leibniz's lead in seeing them as establishing the legitimacy of teleological explanations within the domain of physics. With the explanatory power of principles like the MDPP now being beyond doubt, contemporary philosophers have challenged their status as genuinely teleological. Since concepts change and evolve over time, this might well be taken as simply a shift in our understanding of teleology and teleological explanation. (Clearly the contrast with mechanistic explanations, for example, plays a less significant role in

${ }^{37}$ For general discussions of this development, see Goldstine (1980), Woodhouse (1810), and, especially, Yourgrau and Mandelstam (1968). 
our conception of a teleological explanation today than it did in Leibniz's time.)

Nonetheless, it seems to me as though contemporary explications of teleology have, if anything, tacked towards Leibniz's less Aristotelian conception of teleology than away from it, and that contemporary philosophers have been rather unfair in their offhand dismissals of Leibniz's view. Without hoping, or wishing, to give an exhaustive defense of Leibniz's position on natural teleology, it might therefore be worthwhile to consider two main strategies for resisting Leibniz's position. ${ }^{38}$ Doing so should not only help to further bring out the coherence of Leibniz's view, but also highlight a tension in contemporary accounts of teleological explanations which attempt to defend their legitimacy in domains such as systematics, biology, and cognitive science, while denying that teleological explanations have a role to play within physics itself.

One initially attractive strategy for resisting Leibniz's position is to suggest that genuine teleology requires a thicker conception than the one with which Leibniz works. In particular, one might argue that the concept of teleology requires some reference either to the good or intentionality in order to qualify as satisfactorily robust. While there is no denying that notions of goodness and intentionality are strongly associated with teleological explanations, making reference to them a necessary condition for teleological explanations would, I suggest, lead to serious difficulties that contemporary philosophers of science have been understandably eager to avoid.

\footnotetext{
${ }^{38} \mathrm{~A}$ third strategy would argue that there is something metaphysically incoherent, or at least problematic, in the postulation of teleological laws of nature. This issue is taken up in McDonough 2007. For discussion of some issues raised neither there nor below, see Stöltzner (1994, 1999, and 2000).
} 
Consider first the suggestion that genuine teleology must involve some reference to the good. This suggestion invites two different sorts of objections. The first, and I think lesser, is a range of plausible counterexamples implying that building the concept of the good into the concept of a legitimate teleological explanation would force us to count as non-teleological many things that are generally considered to be teleological. So, to take just one example, it is widely assumed that the behavior of heat-seeking missiles is teleological, even though it is difficult to see - especially given that they explode on impact - how in finding their targets they act for their own good. To this, it might reasonably be suggested that the good referenced to in connection with heat-seeking missiles is derived from their designers or users. But this response invites problems of its own, for it would seem that artifacts might be teleological even if used for purposes other than those for which they were created (a missile used by the home team is no less teleological than a stolen one used by the enemy), and might be teleological even if they undermine the purposes to which they are used (a missile fired by the home team that strikes a friendly plane is no less teleological than one that strikes an enemy plane). The good of the creator, or user, thus seems to have little to do with whether or not the system itself is teleological. ${ }^{39}$ If all of that is right - and no doubt one could wrangle over the details at great length - it would still be possible to suppose that ascriptions of teleology

${ }^{39}$ More fancifully, but perhaps with more conceptual clarity, the same point could be made by imagining the case of a "swamp-missile" formed by a windstorm in a parts factory, which miraculously resembles exactly a human-made missile. Given their identical behaviors, it will be difficult to suppose that one missile, but not the other, is teleological even though only one of the missiles could have possibly derived its good from a creator. 
involve metaphorical or projective goods. But that, of course, is no objection to Leibniz's account of teleology since nothing precludes thinking that in taking the easiest or most determinate path a ray of light acts for a good in such an extended sense.

The second, and I think more pressing, objection to building a concept of the good into the concept of teleology stems from methodological considerations. Most contemporary philosophers working on teleology have tried to get away from building evaluative judgments into the meaning of teleology precisely because it would seem to undermine the scientific legitimacy of teleological explanations. To know that the bear is swatting at the beehive in order to get honey, we had better not have to know that he is acting for The Good or even His Good lest biological descriptions be no more testable, or falsifiable, than the pronouncements of moralists. The point here is perhaps easily overlooked since there need not be anything problematic - except for the confusion that it tempts - about scientific descriptions appealing to, for example, "the good of the organism." The presence of evaluative language in such statements is innocuous insofar as it can be reduced to ultimately non-evaluative terms such as "increases caloric intake" or "is likely to lead to positively differential gene survival" and thus shown not to be essentially evaluative at all. The same, of course, is true for processes studied by physicists: nothing precludes evaluative sounding talk in the description of physical processes as long as such talk can ultimately be eliminated in favor of scientifically confirmable quantities and the like. ${ }^{40}$

The suggestion that teleology requires intentionality likewise runs into two different kinds of objections. First, it similarly - but more importantly this time - faces a

${ }^{40}$ For a recent attempt to defend the centrality of value judgments to teleological explanations see Bedau 1992a and 1992b. 
range of plausible counter-examples. Although it is generally granted that the behavior of relatively primitive organisms can be teleological, most people will be reluctant to ascribe intentionality to them. Plants may grow crooked in order to get more sunlight, ants may march up trees in order to escape high-waters, and dogs may defile fire hydrants in order to mark their territory, but only on the absolutely barest conceptions of intentionality will such behaviors be thought to involve intentionality. (And, of course, on the barest conceptions of intentionality the thesis that teleology must involve intentionality would be no threat to Leibniz's account - if everything from podiums to angels can be seen as having intentionality then having intentionality won't disqualify anything from falling under a teleological description.) Not unrelatedly, philosophers of biology have also been eager to ascribe teleology to well-functioning organs, but again they would clearly be reluctant to ascribe intentionality either to the formation or function of these organized systems. Hearts are thought to pump in order to circulate blood, but not (or at least not any longer) because it is assumed that they were created by an intelligent being.

Second, and perhaps more controversially this time, there are also methodological reasons for resisting the temptation to build intentionality into the concept of teleology. For, on the one hand, one might well think that intentionality is a more complex, less well understood concept than teleology itself, so that leaning on the concept of intentionality would be a step backwards in providing an analysis of teleology. And, on the other hand, one might also well think that our best strategy for giving an illuminating - and naturalistic - account of intentionality is in terms of complex teleological behavior. If 
that is true, defining the latter in terms of former would not merely be a step backwards, but would get the explanatory order exactly the wrong way around. ${ }^{41}$

For both philosophical and naturalistic reasons, contemporary philosophers have therefore typically defended relatively thin conceptions of teleology - that is conceptions which do not make non-eliminable appeals to goods, intentionality, immanent strivings or formal natures. In this way, the conceptions of teleology defended by contemporary philosophers have generally had more in common with the conception that Leibniz employs in his discussion of the laws of optics than with Aristotelian or Scholastic conceptions. Rather than leading to greater sympathy for Leibniz's conclusion that teleological explanations might have an important role to play even in the domain of physics, however, this overlap has instead given birth to a second strategy for resisting his position, namely, arguing that there are principled reasons for rejecting Leibniz's explanations as non-teleological even on a thin conception of teleology. Although this is indeed the most common strategy employed by contemporary philosophers working on the concepts of teleology and teleological explanations, it is far from clear that they have made a convincing case against Leibniz's position. In order to see this more clearly, let's briefly consider three relatively recent attempts to highlight a principled reason for denying that explanations such as those offered by Leibniz are genuinely teleological even on a suitably thin conception of teleology.

(1) It is sometimes implied today that counting variational principles as teleological would massively violate the dictates of commonsense and linguistic intuition. This suggestion has recently been brought out forcefully by a provocative example

\footnotetext{
${ }^{41}$ A helpful overview, as well as a regularly updated bibliography, of such approaches to intentionality is available in Neander 2004.
} 
offered by Jonathan Bennett. After struggling to find any good reason for why Snell's law should be considered genuinely teleological - where Snell's law is understood as "the proposition that light passing between different mediums always follows the easiest path from A to B" - Bennett finally settles on the following consideration:

Perhaps, then, the idea is that Snell's law ... speaks of bodies' behaving in such a way that the resultant situation is thus-and-so. If the body dependably behaves now in a manner that leads to the obtaining of a certain state of affairs later, some kind of forward-lookingness seems to be involved; and this might seem to smack of teleology. But if this is teleology or an appearance of it, the ordinary behaviour of poured water also invites a teleological interpretation: water is poured into one end of a pool, someone draws a bucket of water out of the other end, and a beaver swims across the middle; millions of water molecules variously move in such ways that the final state of the pool's surface is a smooth plane at right angles to the line of gravity. Yet nobody, so far as I know, has claimed to detect a teleological pattern here. (Bennett 2001, 1: 271-2)

The implied argument here seems to be a species of reductio ad absurdum. If we were to grant that principles such as the MDPP were teleological, we should also be forced to grant by parallel reasoning that even the behavior of poured water might be given a genuinely teleological explanation. But something like commonsense, or linguistic intuition, is supposed to show us that that would be absurd, and so, we must deny that principles such as the MDPP are genuinely teleological after all.

Leibniz might, of course, try to respond to such an argument by challenging the purported absurdity of the example. He could, for example, fairly point out that for much of the history of western science the suggestion that the behavior of material stuffs might 
be explained teleologically was not at all considered to be absurd, but rather taken for granted. It is, in fact, only relatively recently that one could even imagine that nobody would claim to detect a teleological pattern in the behavior of poured water, or falling rocks, or rising fire. Indeed, Leibniz might even point out that our contemporary language is still shot through with talk that at least prima facie appears to be teleological: electricity seeking the easiest path, elastic solids trying to regain their original shapes, and, yes, even water finding its lowest resting point. Even if these ways of speaking do not constitute much of an argument to the effect that such processes are genuinely teleological, Leibniz might nonetheless insist that the idea that the behavior of poured water might be explained teleologically is at least not as crazy as Bennett's example seems to imply.

In addition to directly challenging the intuitions on display in Bennett's example, however, Leibniz might also reasonably question the significance of those intuitions. Any interesting theory of teleology will, of course, both have to agree in some measure with our commonsense and linguistic intuitions, and in some measure will have to help clarify, revise, or extend our intuitions. A theory that did not do the former would simply not be a theory about teleology, but rather a theory about something else altogether. One that did not accomplish the latter would not be a theory about teleology either, but rather something more akin to a dictionary definition or a sociological account of how we use words like "teleology" and "teleological." Within these parameters, however, a wide range of approaches is still available. In the shadow of logical positivism, and the socalled "linguistic turn," contemporary philosophers have often fallen on one end of the spectrum, seeking to tailor their accounts rather closely to what they think the person on the street, or perhaps in the laboratory, would already count as teleological. In the wake 
of the scientific revolution, and the development of his own iconoclastic system, however, Leibniz not surprisingly falls on the other end of the spectrum, and is clearly more stimulated by the prospect of developing a bold new set of teleological explanations than in preserving the intuitions of either his scholastic predecessors or his mechanistic contemporaries.

Given Leibniz's more liberal bent, there is therefore no clear reason why he should not simply run an example like Bennett's in the opposite direction. Starting with the premises that (i) the MDPP is a teleological principle, (ii) the behavior of poured water can be explained by appeal to the (so-called) principle of least potential energy, and (iii) the suggestion that consistency requires us to treat that principle and the MDPP on a par, Leibniz might well draw the conclusion that the behavior of poured water can, after all, be explained teleologically. If that new conclusion cuts against some dictates of commonsense, or linguistic intuition, well, then, Leibniz might suppose, so much the worse for commonsense and linguistic intuition. After all, by Leibniz's lights - and by ours! - such intuitions have been at best unreliable friends in the development of a systematic account of the natural world. ${ }^{42}$

(2) A different objection to Leibniz's characterization of variational principles as teleological has been suggested by Ernest Nagel in his influential "system-property" account of natural teleology (Nagel 1961, 398-446, and 1979). According to Nagel, the four following characteristics are essential to all teleological processes. (1) The processes must be plastic in the sense that "the goal of such processes can generally be

${ }^{42}$ The dispute here is, I think, interestingly mirrored in contemporary debates over the legitimate scope of intentional explanations. See, for example, the first two chapters of Dennett (1987), as well as, Bennett (1991). 
reached by the system following alternate paths or starting from different initial positions" (Nagel 1979, 286). (2) A teleological process must be persistent, i.e. it must exhibit "the continued maintenance of the system in its goal-directed behavior, by changes occurring in the system that compensate for any disturbances taking place (provided these are not great) either within or external to the system, disturbances which, were there no compensating changes elsewhere, would prevent the realization of the goal (Nagel 1979, 286). (3) The variables of the processes must be independent or orthogonal to one another so that "apart from those situations in which determinate relations hold between the variables because of their role in goal-directed processes, the known (or assumed) "laws of nature" impose no restrictions on the simultaneous values of the variables" (Nagel 1979, 289). (4) The three preceding conditions must hold not merely for some particular situation or other, but also under a range of counterfactual conditions. A system should not be considered goal-directed if it "just happens" to satisfy the first three conditions. It must also be the case that it would satisfy those conditions if - within certain limits - features of the system or circumstance were altered (Nagel 1979, 287). ${ }^{43}$

${ }^{43}$ In introducing this requirement, Nagel writes, "However, for the process to count as goal-directed on the system-property view, it is not sufficient that on some given occasion (or even on several occasions) the kidneys 'just happen' to eliminate excess water from the blood and so 'happen' to keep constant the concentration of water in it. ... To be goal-directed, the process must satisfy the much stronger requirement that were the blood inundated with water to a greater or lesser extent than was actually the case, the activity of the kidneys ... would have been appropriately modified. What this amounts to is that for each member of a sequence of possible values (within certain limits) of water content of the blood, there is a member of the sequence of possible values of $\mathrm{K}$, such that 
Three of these characteristics present no difficulties for Leibniz's position. A ray of light traveling from A to B via a reflecting or refracting surface, for example, satisfies the requirement of plasticity due to the fact that it may start from a wide range of initial positions, and travel along any number of corresponding paths depending on the relevant medium, while always taking the "most determined path" in Leibniz's technical sense. A ray of light under such circumstances also satisfies the requirement of persistence since modifications of the medium, or surface, will generally be compensated for in such a way that the path of the ray will continue to be the "most determined" between A and B. Furthermore, both of these requirements hold under a suitably wide range of counterfactual conditions - the ray would still take the most determined path if the medium were different or if the starting point had been altered - thereby satisfying what we might call Nagel's requirement of "counterfactual extendibility."

As Nagel is eager to point out, however, the orthogonality condition would brand as non-teleological many systems whose behavior can be readily explained in terms of variational principles. To borrow one of Nagel's own examples, the behavior of a ball in a hemispherical bowl can be explained in terms of the (variational) principle of least potential energy since, if it is displaced from its resting point at the bottom of the bowl, it will act in such a way as to once again minimize (better: "make stationary") its potential energy. The relevant variables involved, however, are not independent in Nagel's technical sense "since the restoring force is proportional to the magnitude of the displacement force, though oppositely directed" (Nagel 1979, 287). Thus, according to Nagel's account, the behavior of the ball will not count as teleological because it fails to for each pair of these corresponding values the goal G would be achieved - that is, for each such pair, the water content of the blood would be 90 percent" (Nagel 1979, 287). 
satisfy his "orthogonality" condition. The same considerations would similarly rule out all of the examples that Leibniz offers as evidence of teleological processes in the domain of physics.

Granting that Nagel's orthogonality condition would deal a devastating blow to Leibniz's account of natural teleology, we might well wonder what reasons Leibniz could be given for accepting it. Setting aside Nagel's occasional appeals to brute intuitions (that straightforwardly beg the question against Leibniz's position) we find a more intriguing line of argument in Nagel's repeated suggestions that at least something like the orthogonality condition must be accepted if the notion of a teleological system is to have any basis at all. In a crucial passage from his seminal piece, "Teleology Revisited," Nagel writes:

I must briefly explain why this [orthogonality] requirement is important: in short, it is this requirement that serves as a formal criterion for distinguishing processes that are goal-directed from those which are commonly held not to be such.... When a ball at rest inside a hemispherical bowl is displaced from its equilibrium position, restoring forces come into play that in the end bring the ball to rest at its initial position. Is this a goal-directed process, whose goal is the restoration of equilibrium? Were such a process so classified, every process in which some equilibrium state is restored would also have to be designated as goal-directed; and in consequence, the designation would be applicable to well-nigh all processes, so that the concept of being goal-directed would not be differentiating, and would therefore be superfluous. (Nagel 1979, 288) 
The same idea is on display in an important passage from the Structure of Science where a pendulum plays the role of a simple variational system rather than a ball in a hemispherical bowl:

In short, the values of these proposed state variables at any given instant are not independent. It therefore follows that the simple pendulum is not a directively organized system in the sense of the definition presented. Moreover, it is also possible to show in a similar manner that a number of other systems, generally regarded as nonteleological ones, fail to satisfy that definition. ... since there are at least some systems not usually characterized as teleological which must also be so characterized [as not teleological] on the basis of the definition, the label of ‘directively organized system’ whose meaning the definition explicates does not apply to everything whatsoever, and it does not baptize a distinction without a difference. $(1961,420)$

Implicit in these comments, I suggest, is the deep reason for Nagel's insistence on the orthogonality condition. Nagel's entire discussion of teleological systems is framed by a concern to distinguish teleological systems from non-teleological systems. Allowing variational principles to serve as foundations for teleological explanations threatens that distinction by implying that almost any - perhaps every - system might qualify as a teleological system. The orthogonality condition - or at least something like it - is thereby seen to be compelling by Nagel's lights if there is to be any point at all in giving an account of teleology and teleological explanations.

So understood, however, Nagel's insistence on the orthogonality condition seems to be merely an artifact of the contemporary concern to demarcate teleological processes and systems from non-teleological processes and systems - an objective that has its roots 
in the rejection of the Aristotelian-Scholastic tradition, but one that is clearly not shared by Leibniz himself. For Leibniz the central question that an account of natural teleology must answer is whether or not teleological explanations can be legitimately employed anywhere within the domain of physics. As a result, the possibility held out by variational principles that every physical event might be given a teleological explanation no more threatens to undermine his objective in investigating natural teleology than the possibility of explaining every physical event in terms of mechanical laws threatens to undermine the coherence of mechanism. Indeed, the prospect of two complete systems of explanation - one teleological, one mechanical - according to which every physical event might in principle be explained either way is one that Leibniz happily embraces. Thus, for example, in the Tentamen Anagogicum, he proudly proclaims that:

I usually say that there are, so to speak, two kingdoms even in corporeal nature, which interpenetrate without confusing or interfering with each other - the realm of power, according to which everything can be explained mechanically by efficient causes, when we have sufficiently penetrated into its interior, and the realm of wisdom, according to which everything can be explained architectonically, so to speak, or by final causes when we have understood its ways sufficiently. (TA 273/L 479)

Although Leibniz was in no position to confidently assert that two such systems of explanation could actually be realized, the development of variational principles in contemporary physics has nonetheless gone a remarkably long way towards vindicating his ambitious hypothesis. I conclude that there is therefore no good reason for Leibniz to accept Nagel's orthogonality condition, and that in the absence of that condition, Nagel's line of objection to Leibnizian natural teleology collapses. 
(3) Finally, it is sometimes suggested that the possibility of reducing the typically integral equations of variational principles to typically differential equations of more familiar "efficient laws" undermines any teleological interpretation of those principles. ${ }^{44}$ The central thrust of this objection can be illustrated in a less technical context by another helpful example introduced by Bennett:

Stable Lake is a body of water occupying an extinct volcanic crater in the Yukon. Around its edges, above the water-line, alpine lilies grow; and rocks beneath the water harbour a population of fresh-water shell-fish. The lake loses water only by evaporation, and gains it only through rainfall. The lilies cannot survive immersion, nor can the shellfish survive exposure to the air ... one may be surprised to learn of evidence that Stable Lake protects its flora and fauna from the disasters I have mentioned. In any normal year, the water-level sometimes nearly reaches the lilies and sometimes falls almost to the level of the shellfish; but in an abnormally rainy year the flowers are still not drowned, nor are the shellfish desiccated in an abnormally dry one. In fact, Stable Lake conforms to two teleological laws: (1) Whenever the lake registers that $\mathrm{f}$ is required and sufficient for not-drowning-the-lilies, it does $\mathrm{f}$ [where f ranges over things that the lake can do]. (2) Whenever the lake registers that $\mathrm{f}$ is required and sufficient for not-desiccating-the-shellfish, it does f. Combining the two: whenever the lake

${ }^{44} \mathrm{Cf}$ : "The equivalence of a variational principle to its corresponding set of Euler equations is also sometimes regarded as an argument against interpreting differential equations as indicative of cause and integral principles as attesting purpose, since the transformation from the integral to the differential form would then amount, as Margenau puts it, to transmuting a purpose into a cause" (Yourgrau and Mandelstam 1968, 176). 
registers that $\mathrm{f}$ is required and sufficient for saving-the-wildlife, it does $\mathrm{f}^{45}$ (Bennett 1976, 75-76)

Bennett, like Leibniz, maintains that teleological explanations do not presuppose intentional entities at the level of physics. He therefore rejects the suggestion that a teleological explanation of the lake's behavior can be ruled out simply because the lake lacks intentions and desires (Bennett 1976, 76). Again, like Leibniz, Bennett also believes that the behavior of a particular system or process might be explained both in teleological and non-teleological terms (Bennett 1991, 178). He therefore also denies that a teleological explanation of the lake can be ruled out by the fact that its behavior can be given a non-teleological explanation (Bennett 1976, 77).

Nonetheless, Bennett maintains that a teleological explanation in the case of Stable Lake would clearly be "fraudulent." He locates the difficulty in the fact that "a single mechanistic explanation [that relates the surface area of the lake to the rate of evaporation] covers exactly what the teleological explanation covers." In these circumstances, Bennett reasons, the proposed teleological explanation is disqualified by the presence of its non-teleological counterpart because no justification can be given for using the teleological explanation rather than the non-teleological explanation (Bennett

\footnotetext{
${ }^{45}$ I have modified Bennett's example slightly by replacing some technical notation introduced earlier in his chapter with its non-technical equivalent as described on his page 76. Perhaps, it should also be noted that the term "registers" is generally used in a technical sense by Bennett in (1976), but that - as he points out - registration in that sense is idle in the Stable Lake example.
} 
1976, 77; 2005, 145-6). ${ }^{46}$ Following this line of thought, one might suppose that the same lesson should be applied in the case of variational principles. Given that explanations in terms of variational principles can generally be directly translated into explanations in terms of non-variational principles (or "laws"), ${ }^{47}$ one might conclude that the former are similarly disqualified by the presence of the latter.

Here once again Leibniz might well argue the relevant facts of the matter. He could fairly insist that even granting the direct translatability of variational principles into non-variational laws, the use of the former is nonetheless justified for two important reasons. First, the equations that result from translating variational principles into nonvariational laws are frequently more cumbersome and difficult to manipulate. Indeed, in

46 "That mechanistic explanation disqualifies the teleological one - not because each thing Stable Lake does can be explained mechanistically, but because a single mechanistic explanation covers exactly what the teleological explanation covers. We do not even have a faute de mieux defense of the latter, for the mechanistic explanation is known and is easy to apply: it is no harder to notice that the lake's surface-area has altered than to notice that if the rate of evaporation does not alter the wildlife will suffer" (Bennett 1976, 77).

47 "As a matter of fact, most sets of (mathematically) simple differential equations can be so formulated [as variational principles]. Conversely, any variational principle is equivalent to a set of differential equations. It follows immediately that once the laws of a physical theory are expressed as differential equations, the possibility of their reduction to a variational principle is evident from purely mathematical reasoning and does not depend on certain attributes intrinsic to the theory" (Yourgrau and Mandelstam 1968, 176). 
their classic discussion of the topic, Wolfgang Yourgrau and Stanley Mandelstam certainly no friends of teleological interpretations of variational principles - concede that "This possibility [of reducing differential equations of physical laws to integral equations of variational principles] is of pragmatic value ... for, other factors aside, ... it allows the equations to be written in such a way as to be independent of the co-ordinate system. In addition, the transformation of the equations to the Hamiltonian [i.e. variational] form is vitally important for quantum mechanics" (Yourgrau and Mandelstam 1968, 176). Second, in addition to citing their computational usefulness, Leibniz could point out that variational principles earn their keep by highlighting, in at least two different ways, common patterns in otherwise disparate physical phenomena: ${ }^{48}$ (i) they display a single mathematical form running implicitly through many of the most important equations of classical and contemporary physics; ${ }^{49}$ (ii) they often allow the unification of several different laws - both variational and non-variational - to be subsumed under a single unifying principle. Newton's second law, the Principle of Least Action, and the Principle of Least Potential Energy, for example, can all be treated as special cases of Hamilton's (Extended) Principle (Lemons 1997, 102).

These considerations suggest that the use of variational principles is not otiose given their non-variational counterparts, and thus that their use may be justified on ${ }^{48}$ Cf. Bennett's "unity condition” as a requirement of intentionality (Bennett 1991, 177). ${ }^{49}$ Lemons, for example, notes that "Given appropriate variational principles each with an associated multiple integral and scalar integrand, we can produce all the important partial differential equations of physics: the wave equation, the diffusion equation, Poisson's equation, Schrodinger's equation, and each of Maxwell's equations" (Lemons 1997, 111). 
pragmatic grounds. But Leibniz could also challenge the lesson Bennett draws from the Stable Lake example. Suppose that the behavior of a particular system could be accounted for both in terms of variational principles and non-variational laws. Suppose further that in some particular case the account in terms of variational principles is both mathematically less elegant and so singular as to provide little help in bringing out similarities between the phenomena under investigation and other physical phenomena. Under such circumstances, all parties could presumably agree that an account in terms of variational principles might well be unhelpful for practical purposes. But having granted that the account in terms of variational principles is less useful, why should they go on to suppose that it is "fraudulent?" To do so would seem to build not just practical utility, but greater practical utility than all possible rivals into the very meaning of "teleological explanation.” But Leibniz might well argue that that would be special pleading. After all, no one, I take it, would suppose that with roles reversed - so that a variational account were more useful than its mechanical counterpart - the mechanical account would thereby be shown to be counterfeit. ${ }^{50}$ Leibniz thus seems within his rights in

${ }^{50}$ It is important not to confuse the objection being considered here - that the presence of suitable non-teleological explanations ipso facto undermines the legitimacy of otherwise acceptable teleological explanations - with the more metaphysical objection that the interderivability of modern variational principles and more familiar "efficient laws" collapses any significant distinction between the two. Even if successful, the metaphysical objection would not impugn Leibniz's teleological characterization of principles like the MDPP (what is at stake here), but would instead suggest that the development of physics has shown that both "principles" and "laws" need not be - or perhaps cannot be - counted as explanatory primitives. I argue elsewhere that Leibniz's 
insisting that the legitimacy of teleological explanations need not be undermined by the mere availability of equivalent non-teleological explanations.

Finally, it should be added that Leibniz need not embrace the assumption that a teleological account must be more useful than a substitutable mechanical account in order to reject the particular teleological explanation offered of Stable Lake's behavior. For in that example, the lake's doing what is required and sufficient for saving-the-wildlife seems to be merely a coincidental feature of the set-up as witnessed by the fact that if the wildlife were simply destroyed the behavior of the lake would remain exactly the same. The modest hypothesis that the behavior of a genuinely teleological system must be responsive to its purported goal(s) is therefore sufficient for discounting the proffered teleological account of the lake's behavior. And this is a lesson that Leibniz could easily embrace as the examples he prefers are in fact at least minimally responsive in just this way. A freely hanging chain, for example, which has some of its links replaced by heavier, lighter, or more elastic links will hang differently than it did before, but will nonetheless still maximize its total possible descent.

\section{Conclusion}

own account of the laws of nature - according to which the laws of nature are abstractions from more fundamental derivative forces - affords him the resources for resisting the collapse objection. The issues that objection raises within context of Leibniz's system, however, are rather far reaching and deserve more extensive treatment than can be offered here. For further discussion of the metaphysics of Leibniz's two realms doctrine as applied to the laws of nature, see my (2007). 
Leibniz's view that teleological explanations may play an important role within the domain of physics is as unpopular today as it was in his own time. Careful consideration of his work in optics, and the notion of natural teleology which that work supports, however, suggests that the strengths of Leibniz's position have been greatly underestimated. Laws like the MDPP, whose discovery and formulation Leibniz helped to pioneer, represent an enduring, mathematically precise, and empirically confirmable alternative to the mechanistic explanations of his contemporaries, while his teleological interpretation of those laws continues to challenge widely accepted views concerning the nature and role of teleological explanations in modern physics. Far from constituting an embarrassment to his mature natural philosophy, or an opportunistic concession to his predecessors, Leibniz's defense of natural teleology represents one of his most profound attempts to reshape the scientific framework that was emerging in throes of the scientific revolution. 


\section{Bibliography}

Abra de Raconis, C. F. 1651. Tertia pars philosophiae, seu physica (Lyons: Irenaei Barlet). (Original publication: 1617, with twelve subsequent editions, mostly in Paris, through 1651.)

Bacon, Francis. 1858. Works, edited by James Spedding, Robert Leslie Ellis, and Douglas Denon Heath (London: Longman). (Facsimile reprint, Stuttgart-Bad Cannstatt: Friedrich Frommann, 1963.) Reference is to volume and page.

Bedau, Mark. 1992a. "Where's the Good in Teleology?" Philosophy and Phenomenological Research (52) 781-806. Reprinted in Colin Allen, Marc Bekoff, George Lauder, eds., Nature's Purposes: Analyses of Function and Design in Biology (Cambridge: MIT Press) 261-291.

Bedau, Mark. 1992b. "Goal Directed Systems and the Good,” The Monist (75) 34-49.

Bennett, Jonathan. 1976. Linguistic Behaviour (Cambridge: Cambridge University Press). Reissued by Hackett Publishing Company: Indianapolis, 1989.

-----. 1983. "Teleology and Spinoza's Conatus," in Midwest Studies in Philosophy, Volume VIII: Contemporary Perspectives on the History of Philosophy, 143-160.

-----. 1984. A Study of Spinoza's Ethics (Indianapolis: Hackett Publishing).

-----. 1990. "Spinoza and Teleology: A Reply to Curley," in Spinoza: Issues and Directions, edited by Edwin Curley and Pierre-Francois Moreau (Leiden: E. J. Brill).

-----. 1991. "Folk-Psychological Explanations," in The Future of Folk Psychology, edited by John D. Greenwood (Cambridge: Cambridge University Press).

-----. 2001. Learning from Six Philosophers, in two volumes (Oxford: Oxford University Press). Reference is to volume and page.

----. 2005. "Leibniz's Two Realms," in J. A. Cover and D. Rutherford (eds.), Leibniz, Nature and Freedom (New York: Oxford University Press).

Bernstein, Howard. 1984. "Leibniz and Huygens on the 'Relativity' of Motion." Studia Leibnitiana (13) 97-113.

Cooper, John. 1987. Hypothetical Necessity and Natural Teleology, in Philosophical Issues in Aristotle's Biology, edited by A. Gotthelf and J. B. Lennox (New York: Cambridge University Press).

Curley, Edwin. 1990. “On Bennett's Spinoza: The Issue of Teleology,” in Spinoza: Issues and Directions edited by Edwin Curley and Pierre-Francois Moreau (Leiden: E. J. Brill) 39-52.

Dennett, Daniel. 1987. The Intentional Stance (Cambridge, Massachusetts: The MIT Press).

Della Rocca, Michael. 1996. "Spinoza's Metaphysical Psychology," in The Cambridge Companion to Spinoza, edited by Don Garrett (Cambridge: Cambridge University 
Press).

Descartes, René. 1897-1913. Oeuvres de Descartes, volumes 1-12, edited by Ch. Adam and P. Tannery, reprinted (Paris: J. Vrin, 1957-).

-----. 1965. Descartes: Discourse on Method, Optics, Geometry and Meteorology, translated and edited by P. J. Olscamp (Indianapolis: Bobbs-Merrill).

-----. 1985. The Philosophical Writings of Descartes, volumes 1-2, translated and edited by J. Cottingham, R. Stoothoff, D. Murdoch (Cambridge: Cambridge University Press).

Des Chene, Dennis. 1996. Physiologia: Natural Philosophy in Late Aristotelian and Cartesian Thought (Ithaca: Cornell University Press).

Fellmann E. A. and J. O. Fleckenstein. 1970-1980. "Bernoulli, Johann" in Dictionary of Scientific Biography, edited by Charles Coulston Gillispie (New York: Scribner).

Fermat, Pierre. 1891-1912. Oeuvres de Fermat, four volumes, edited by P. Tannery and Charles Henry (Paris).

Gabbey, Alan. 1980. "Force and Inertia in the Seventeenth Century: Descartes and Newton," in Descartes: Philosophy, Mathematics and Physics, edited by Stephen Gaukroger (Sussex: Harvester Press) 230-320.

Garrett, Don. 1999. "Teleology in Spinoza and Early Modern Rationalism," in New Essays on the Rationalists, edited by Rocco J. Gennaro and Charles Huenemann (New York: Oxford University Press).

Goldstine, Herman. 1980. A History of the Calculus of Variations from the $17^{\text {th }}$ through the $19^{\text {th }}$ Century (New York: Springer-Verlag).

Herons von Alexandria. 1900. Mechanik und Katoptrik, Edited and translated into German by von L. Nix and W. Schmidt from Heronis Alexandrini Opera quae supersunt omnia, volume 2, facsimile: (I. Leipzig).

Hildebrandt, Stefan and Anthony Tromba. 1985. Mathematics and Optimal Form (New York: Scientific American Books).

Hofmann. J. E. 1970-1980. "Bernoulli, Jakob" in Dictionary of Scientific Biography, edited by Charles Coulston Gillispie (New York: Scribner).

Hull, D. 1981. "Philosophy and Biology," in G. Fløistad (ed.) Contemporary Philosophy: A New Survey, volume II (The Hague) 281-316.

Huygens, Christiaan. 1888-1950. Oeuvres completes de Christiaan Huygens, 22 volumes (La Haye: Société Hollandaise des Sciences). Reference is to volume and page.

Korteweg, D. J. 1896. “Descartes et les manuscrits de Snellius d'après quelques 
documents nouveaux.” Revue de métaphysique et de morale (IV) 489-501.

Leibniz, G. W. 1678 (- 1680/81?). "Definitiones Cogitationesque Metaphysicae," in A VI, 4B, 1393-1405.

-----. 1682. "Unicum Opticae, Catoptricae \& Dioptricae Principium," Acta Eruditorum, June. Reprinted in Acta Eruditorum, volume 1 (New York: Johnson Reprint Corporation).

----. 1686. "Discourse on Metaphysics," in G 2:427-63. Reference is by section.

----. 1696. "Tentamen Anagogicum: An Anagogical Essay in the Investigation of Causes," in G 7:270-79.

----. 1695b. "A Specimen of Dynamics," in GM 6: 235-54.

----. 1697. "On the Ultimate Origination of Things," in G 7:302-8.

----. 1697b. "Résumé of Metaphysics," in G 7:289-92. Reference is to section number.

-----. 1875-90. Die Philosophischen Schriften von Leibniz, 7 volumes, edited by C. I.

Gerhardt (Berlin: Weidmann; reprinted Hildesheim: Georg Olms, 1978).

---- 1923-present. G. W. Leibniz: Samtliche Schriften und Briefe, edited by German Academy of Sciences (Berlin: Akademie Verlag). References are to series, volume and page.

----. 1969. G. W. Leibniz: Philosophical Papers and Letters, $2^{\text {nd }}$ ed., translated and edited by L. E. Loemker (Dordrecht: Reidel).

----. 1989. G. W. Leibniz: Philosophical Essays, translated and edited by R. Ariew and

D. Garber (Indianapolis: Hackett).

----. 1998. G. W. Leibniz: Philosophical Texts, translated and edited by R. Franks and

R. Woolhouse (New York: Oxford University Press).

-----. 2001. The Labyrinth of the Continuum, Writings on the Continuum Problem, 1672-

1686, translated and edited by Richard T. Arthur (New Haven: Yale University Press).

Lemons, Don. 1997. Perfect Form: Variational Principles, Methods, and Applications in Elementary Physics (Princeton, New Jersey: Princeton University Press).

Mach, Ernst. 1989. The Science of Mechanics, translated by Thomas J. McCormack (La Salle, Illinois: The Open Court Publishing Company).

Maupertuis, P. 1698-1759. Oeuvres de Maupertuis (Lyons: Jean-Marie Bruyset). Reference to volume and page.

McDonough, Jeffrey. 2004. Leibniz on Teleology and the Laws of Optics (Doctoral dissertation, University of California, Irvine).

-----. 2007. "Leibniz's Two Realms Revisited,” Nôus, forthcoming.

Nagel, E. 1961. The Structure of Science (New York: Harcourt, Brace and World).

---- 1979. "Teleology Revisted," reprinted in Teleology Revisited and Other Essays in the Philosophy of Science (New York: Columbia University Press).

Neander, Karen. 2004. "Teleological Theories of Mental Content", The Stanford Encyclopedia of Philosophy (Summer 2004 Edition), Edward N. Zalta (ed.), URL = $<$ http://plato.stanford.edu/archives/sum2004/entries/content-teleological/>. 
Newton, Isaac. 1959. The Correspondence of Isaac Newton, edited by H. W. Turnbull, (Cambridge: Cambridge University Press). Reference is to volume and page.

Rice, L. C. 1985. "Spinoza, Bennett, and Teleology," The Southern Journal of Philosophy (23:2) 241-251.

Ronchi, Vasco. 1970. The Nature of Light: An Historical Survey, Translated by V. Barocas (Cambridge, Massachusetts: Harvard University Press).

Sabra, A. I. 1967. Theories of Light from Descartes to Newton (London: Olbourne).

Simmons, Alison. 2001. "Sensible Ends: Latent Teleology in Descartes' Account of Sensation," Journal of the History of Philosophy (39: 1) 49-75.

Stein, H. 1977. "Some Philosophical Prehistory of General Relativity," in Foundations of Space-Time Theories, edited by J. Earman, C. Glymour, and J. Stachel, Minnesota Studies in the Philosophy of Science, Volume VIII (Minneapolis: University of Minnesota Press) 3-49.

Stöltzner, Michael. 1994. "Action Principles and Teleology," in Inside Versus Outside, edited by Harald Atmanspracher and Gerhard Dalenoort (New York: Springer) 33-62.

----.. 1999. "To What Extent Does Formal Teleology Still Make Sense," in Hermeneutics and Science, edited by M. Féhér, L. Ropolyi, and O. Kiss (Dordrecht: Kluwer) 227-246.

-----. 2000. "Le Principe de Moindre Action et les Trois Ordres de la Téléologie Formelle dans la Physique," Archives de Philosophie (63) 621-655.

Vossius, Isaac. 1662. De lucis natura et proprietate.

Westfall, Richard S. 1986. Never at Rest: A Biography of Isaac Newton (New York: Cambridge University Press).

Woodhouse, Robert. 1810. A History of the Calculus of Variations in the Eighteenth Century (New York: Chelsea Publishing Company).

Yourgrau, Wolfgang and Stanley Mandelstam. 1968. Variational Principles in Dynamics and Quantum Theory, Third Edition (London: Sir Isaac Pitman and Sons). 